\begin{tabular}{l|c|c}
\hline \hline Vol. 26(4):619-633 & Ocean and Polar Research & December 2004 \\
\hline \hline
\end{tabular}

Article

\title{
Composition, Structure and Some Distribution Features of Fouling Community in the Water Intake Tunnel of Vladivostok Heat and Power Plant
}

\author{
Alexander V. Moshchenko and Alexander Yu. Zvyagintsev* \\ Institute of Marine Biology, Far East Branch of Russian Academy of Science \\ Vladivostok 690041, Russia
}

\begin{abstract}
The composition, structure and some of the features of spatial distribution of fouling communities in the cooling system tunnels of Heat and Power Station, Vladivostok City were determined. Three groups of animals, possessing features expressive of topological differentiation as well as categorical differences based on dominant and subdominant species, were clearly distinguished in the fouling community of the water intake tunnel. These are Jassa marmorata on the tunnel vault, Balanus rostratus on the walls, and Mytilus trossulus on the bottom. The group from the tunnel vault should be clearly related to "physically controlled" communities, whereas the groups from the walls and bottom, being also physically controlled, show many of the features of "biologically balanced" communities. Thermal treatment of tunnels, performed in summertime, is grossly inefficient, since mytilids continue to settle in SeptemberNovember as well. In order to prevent intensive settlement of Mytilus trossulus larvae it is recommended that the water intake tunnels integral to the cooling system be placed deeper than $15 \mathrm{~m}$.
\end{abstract}

Key words : fouling communities, Jassa marmorata, Balanus rostratus, Mytilus trossulus,water intake tunnel

\section{Introduction}

Water supply systems of industrial and power enterprises are related to the VI types of artificial substrates (Reznichenko 1978), including absolutely stationary installations, which are situated mainly on land several kilometers from the water reservoir. The starting point for cooling systems is at the sea bottom, but their main interaction with the coastal pelagic layer occurs usually within the limits of $5 \mathrm{~m}$. Less than $0.2 \%$ of the total area of artificial substrates of the World Ocean falls on type VI, but biological impairment of these types of substrates from fouling and related material damage is substantial.

Conditions in seawater canals differ from that of natural ones by higher current velocity, which excludes the possibility of existence of some foulers, and at the same time facilitates growth and development of the other species (barnacles, hydroids, mussels). High current velocity can prevent settle-

*Corresponding author. E-mail : azvyagin@imb.dvo.ru ment of larvae and existence of motile animals, which need a suitable biotope of developed fouling. Current velocity determines not only qualitative composition, but fouling degree as well. For example, in the Newfoundland sea canal, the most abundant fouling was observed at current velocity about $20 \mathrm{~m} / \mathrm{min}$., while at less than $10 \mathrm{~m} / \mathrm{min}$., at $40 \mathrm{~m} / \mathrm{min}$., and also at intermittent water supply it was not registered (Sergy and Evans 1975). Colonial organisms-bryozoa, hydroids and ascidians-always dwell in seawater canals. For them, settlement of one larva is quite sufficient for developing a large colony later on. In freshwater canals, bivalvia usually dominate. Zevina (1994) has reported Dreissena sp. dominated in Europe, Limnoperna fortuneiin Hong Kong and South China, Corbicula manilensis-in China, exotic Asian species Corbicula flunimea-in the USA. In seawater canals of temperate zone, mussels dominate in year-round fouling.

In the European part of Russia, fouling of power plant cooling water systems is studied rather intensively (Turpaeva 1987; Protasov et al. 1991; Zevina 1994). As for the Far 
Eastern part of Russia, this problem is still a blank spot. Only one work, devoted to the role of amphipods has been published (Zvyagintsev and Budnikova 2003). In Japan, scores of power plants have cooling systems with seawater, and biologists exercise permanent supervision over the process of fouling and develop corresponding recommendations on its prevention (Kiyono 2003; Sakaguchi 2003). As for the Vladivostok Heat and Power Plant, corresponding works have not been conducted up to the present. Besides, we are not aware of any special investigations pertaining to the composition and distribution of fouling communities on the internal surface of cooling system pipes. The purpose of this work is to investigate the composition, structure and distribution of fouling in cooling system tunnels of the Vladivostok Heat and Power Plant.

\section{Materials and methods}

Tunnels beds are related to a non-prismatic type because of the geometric changes along the bed length, which concern particularly the profile diameter (Konstantinov et al. 1987). Also, they have an adverse, though insignificant, inclination, and irregular shape: the profile is circular, but the full diameter is not filled. The examined parts of the tunnels begin with an opening of a steel pipe (diameter $1.6 \mathrm{~m}$ ), steeply descending into a pump station. Then the tunnels expand up to $2 \mathrm{~m}$, smoothly turning $45^{\circ}$ counterclockwise, proceeding from the south to the north-northwest (deviation to the west is about $15^{\circ}$ ) for the space of more than $800 \mathrm{~m}$,

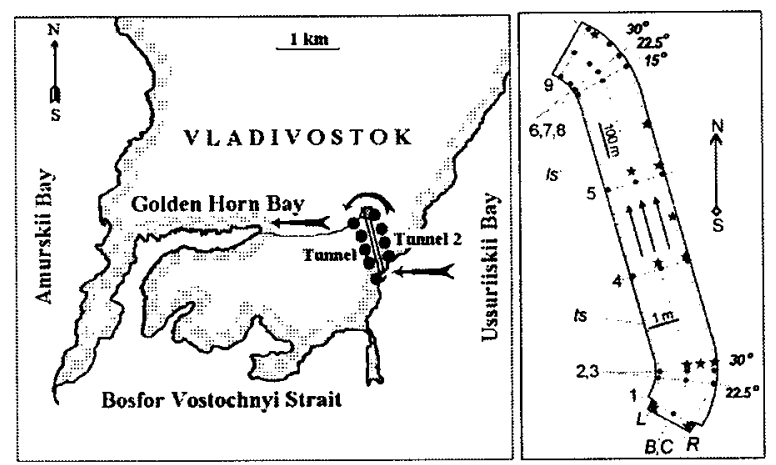

Fig. 1. Location scheme and plan of the tunnel and sampling sites in tunnel 2: numerals $1,2,3, \ldots 9$ designate numbers for cross-sections; letters $L, R, B$ and $C$ - lengthwise sections along the left and right walls, bottom and vault of the tunnel, respectively; arrows - direction of water movement; $l s$ and $t s$ - scale rules for lengthwise and cross measurements of the tunnel, respectively; asterisks sampling sites in three replications in tunnel 1. and again making a $45^{\circ}$ turn to the west, coming over to the distribution cooling system of the Heat and Power Plant (Fig. 1).

Fouling samples were collected after water discharge using a $0.01 \mathrm{~m}^{2}$ sampling square in April 2001. As the period of work was limited by the duration of water discharge and did not exceed 24 hours, with the purpose of determining the convergence of the obtained data, in the first tunnel samples were taken in three replications in the beginning, in the middle and in the end of the tunnel on its vault, bottom, right and left walls (Fig. 1). In the second tunnel, in order to investigate the variations of the quantitative characteristics and fouling composition along the tunnel bed, one sample was taken at each of the 9 sections (designated by roman numerals) on the same levels (literal designations, Fig. 1).

The optimal size of the sampling square, reliably characterizing quantitative parameters of animals and plants, was experimentally grounded by Propp (1971), who considers that a frame is most suitable for use when from 1 to 10 , i.e., 5-6 on the average, animal individuals of every species enter into it. In our case, a $0.01 \mathrm{~m}^{2}$ sampling square turned out to be an optimal frame for fouling communities. Several tens of barnacles, mussels and amphipods can usually be found in such an area. As proved by our experience of long-term observations, the obtained data were sufficiently representational. The variations usually did not exceed $15-$ $25 \%$ of the mean value in the case of three replicated samples. Standard error for the overwhelming majority of samples, taken from tunnel \# 1 , had the same values (Table 1).

Algae and animals collected were identified according to species level, weighed up to within $0.001 \mathrm{~g}$ of wet weight, while population density was calculated for solitary animals. To identify a community we used biomass (B), population density $(\mathrm{A})$, frequency $(\mathrm{F})$, and derivative indices $\sqrt{F \times B}$ and $\sqrt{A \times B}$. To evaluate the distribution pattern and obtain a measure for the aggregation degree of animal populations we used a dispersion index (Elliot 1977; Maximovich and Pogrebov 1986): $I_{w}=\sigma^{2} / \bar{X}$, where $\bar{X}$ is an average population density of each solitary animal, and $\sigma^{2}$ is a variance of their population density for whole samples collected. In random distribution, $I_{w}=1 ; I_{w}>1$ indicates a tendency toward aggregation, while $I_{w}<1$ points to a regular distribution. The significance of the differences of $I_{w}$ from 1 was checked using the statistics $d=\sqrt{2 \chi^{2}}+$ $\sqrt{2 v-1}$, where $\chi^{2}$ (chi-square) is a goodness-of-fit criterion, and $v$ is the number representing the degree of freedom. To obtain a parameter describing the distribution pattern 
Table 1. Mean values (M) and standard error (SE) of biomass of some mass fouling species in tunnel \# 1 from different samples, taken in three replications.

\begin{tabular}{|c|c|c|c|c|c|c|c|c|c|}
\hline \multirow{2}{*}{ Taxon } & \multicolumn{3}{|c|}{ Sample 1} & \multicolumn{3}{|c|}{ Sample 2} & \multicolumn{3}{|c|}{ Sample 3} \\
\hline & $\mathbf{M}$ & $\mathrm{SE}$ & $\% \mathrm{SE}$ & $\mathbf{M}$ & SE & $\% \mathrm{SE}$ & $\mathbf{M}$ & SE & $\% \mathrm{SE}$ \\
\hline Balanus rostratus & 1360 & 92 & 6.8 & 843 & 118 & 14.0 & 516 & 60 & 11.6 \\
\hline Jassa marmorata & 273 & 12 & 4.4 & 293 & 23 & 7.9 & 1989 & 160 & 8.0 \\
\hline Caprellidae & 106 & 17 & 16.0 & 410 & 78 & 19.0 & 16 & 4 & 25.0 \\
\hline Mytilus trosstulus & 18333 & 1590 & 8.8 & 12800 & 901 & 7.0 & 813 & 177 & 21.8 \\
\hline
\end{tabular}

of foulers biomass, an index, similar to the dispersion index was calculated: $I_{w b}=\sigma^{2} / \bar{X}$, where $\bar{X}$ is a mean biomass of animals, including colonial forms, and $\sigma^{2}$ is the biomass variance.

During statistical treatment, we used standard procedures and tests offered by "STATISTICA 5.1" software (Borovikov and Borovikov 1998). To distinguish various groups and associations from one another, the methods of cluster and factor analysis were applied. To simplify the factor structure and to derive easily interpreted solutions, different methods of axes rotation, the varimax, the quartimax, etc. were used (Afifi and Eisen 1982; Kim et al. 1989; Nosov 1990). Selection of number factors was made, following the postulate of parsimony, on the basis of Kaiser criterion and the results of the scree plot test. According to the principle of parsimony, from two competitive solutions, the model, including a minimal number of common factors, is accepted; according to the Kaiser criterion, only the factors with eigenvalues $>1$ are accepted. Application of the scree plot test purports the acceptation of the factor number falling at the area of the curve maximal flattening. Also, to determine the factor number, we use the statistical goodnessof-fit test, if a selected factor solution obtained by the principal components' method and that revealed by the maximum likelihood coincided in terms of the main features.

Application of $\chi^{2}$ criterion permits one to form statistical conclusions about the number of distinguished factors and, in such a way, introduce elements of confirmative factor analysis into the obtained results. Using the method of maximal likelihood, we tested the models, while successively increasing the number of factors and selected the most plausible solution, i.e., such a solution when the probability for the likelihood of the accepted number of factors reached a maximum, or such a solution, when this probability began to exceed the first threshold level $(0.05)$ of validity for a null hypothesis about the absence of distinctions in residual correlation and diagonal matrices (significant correlations between the measured variables are absent). Factor orthogonality was tested by a hierarchical analysis of the oblique factors.

\section{Results}

Visual examination of the tunnels showed that some druses of the Pacific mussel Mytulis trossulus appear in the upper third of tunnels in shelters on the bottom. In the middle of the tunnels, the number of mussel druses increased, and in the lower third, mussels covered the bottom entirely, and to a lesser degree on the sidewalls. Abundant Cirripedia Balanus rostratus, being a perfect substrate for attachment of Amphipoda Jassa marmorata houses and a shelter for other motile benthic forms were found on sidewalls of the tunnels. On the whole, in the most thoroughly investigated tunnel 2 (further-tunnel) 62 animals species were found, including 18 species on the vault, 41 species on the walls (30-on the left wall, and 40-on the right one) and 42 species on the bottom. At that, average fouling biomass reaches $2788.1 \pm 951.6 \mathrm{~g} / \mathrm{m}^{2}$, greatly varying in different tunnel parts (variability range $2.1-28015.6 \mathrm{~g} / \mathrm{m}^{2}$ ).

$M$. trossulus dominates in the tunnel community by biomass and $\sqrt{F \times B}$ index, by frequency-J. marmorata; $B$. rostratus occupies the second place by biomass and $\sqrt{F \times B}$ index, and by frequency, it occupies forth place with $J$. marmorata and hydroid Obelia longissima coming first (Table 2). Thus, taking into account these notes and average population density (1113.9, 1905.6 and 2030.6 ind./ $\mathrm{m}^{2}$, correspondingly for mussels, amphipods and barnacles), this community should be designated as Mytulis trossulus + Balanus rostratus + Jassa marmorata, and if another derived index $\sqrt{A \times B}$-is taken into consideration (its values for the mentioned species are 1520.7, 1125.4 and 160.4 , correspondingly), as Mytulis trossulus + Balanus rostratus.

The total biomass and number of species vary greatly both along the tunnel and by its perimeter. Maximal values of the mentioned parameters were registered on the first turn of the tunnel and immediately after it (sections 2 and 3 ), falling on the bottom and right wall, though by the average number of species, the bottom, right and left walls of the tunnel were almost the same (Figs. 2, 3). The biomass absolute maximum was observed on the bottom of the 
Table 2. Some quantitative characteristics of fouling organisms from the water intake tunnel \# 2 of the Vladivostok Heat and Power Plant.

\begin{tabular}{|c|c|c|c|c|c|c|c|c|c|c|c|c|c|c|}
\hline \multirow{2}{*}{ pecies } & & \multirow{2}{*}{$\sqrt{F \times B}$} & \multirow{2}{*}{$\sqrt{A \times B}$} & \multicolumn{5}{|c|}{ Biomass, $\mathrm{g} / \mathrm{m}^{2}$} & \multicolumn{5}{|c|}{ Population density, ind $/ \mathrm{m}^{2}$} & \\
\hline & & & & $\overline{\bar{X}}$ & $S E$ & $M I N$ & $M A X$ & $I_{W B}$ & $\overline{\boldsymbol{X}}$ & $S E$ & $M I N$ & $M A X$ & $\boldsymbol{I}_{W}$ & \\
\hline 1 & 2 & 3 & 4 & 5 & 6 & 7 & 8 & 9 & 10 & 11 & 12 & 13 & 14 & 15 \\
\hline \multicolumn{15}{|c|}{ Tunnel as a whole } \\
\hline Obelia longissima & 63.9 & 21.32 & - & 7.1 & 5.8 & 0.0 & 210.0 & 171.0 & - & - & $\cdot$ & - & - & -0.017 \\
\hline Halosydna hrevisetosa & 5.6 & 0.40 & - & 0.0 & 0.0 & 0.0 & 1.0 & 1.0 & - & - & - & - & - & 0.394 \\
\hline Hydroides ezoensis & 52.8 & 13.87 & 16.74 & 3.6 & 1.0 & 0.0 & 20.0 & 9.0 & 77.8 & 17.4 & 0.0 & 400.0 & 140.3 & 0.086 \\
\hline Potamilla sp. & 50.0 & 15.60 & 22.75 & 4.9 & 1.4 & 0.0 & 30.0 & 14.0 & 105.6 & 22.2 & 0.0 & 500.0 & 167.5 & -0.020 \\
\hline Nereis multignatha & 13.9 & 2.33 & 2.58 & 0.4 & 0.3 & 0.0 & 10.0 & 7.3 & 16.7 & 7.5 & 0.0 & 200.0 & 120.0 & -0.016 \\
\hline Nereis zonata & 5.6 & 0.39 & 0.00 & 0.0 & 0.0 & 0.0 & 1.0 & 1.0 & 2.8 & 2.8 & 0.0 & 100.0 & 100.0 & 0.001 \\
\hline & 11.1 & 0.99 & 1.18 & 0.1 & 0.1 & 0.0 & 2.0 & 1.5 & 13.9 & 9.0 & 0.0 & 300.0 & 212.0 & 0.201 \\
\hline pira alveolata & 2.8 & 0.28 & 0.00 & 0.0 & 0.0 & 0.0 & 1.0 & 1.0 & 2.8 & 2.8 & 0.0 & 100.0 & 100.0 & -0.081 \\
\hline Typosyllis orientalis & 2.8 & 0.09 & 0.00 & 0.0 & 0.0 & 0.0 & 0.1 & 0.1 & 2.8 & 2.8 & 0.0 & 100.0 & 100.0 & 0.394 \\
\hline Balanus rostratus & 58.3 & 190.75 & 1125.38 & 623.7 & 155.5 & 0.0 & 2650.0 & 1396.5 & 2030.6 & 546.0 & 0.0 & 14000.0 & 5284.9 & -0.020 \\
\hline Balanus improvisus & 38.9 & 23.24 & 49.32 & 13.9 & 5.8 & 0.0 & 160.0 & 87.9 & 175.0 & 39.9 & 0.0 & 800.0 & 327.8 & -0.072 \\
\hline Balanus crenatus & 13.9 & 8.72 & 9.58 & 5.5 & 4.5 & 0.0 & 160.0 & 130.7 & 16.7 & 7.5 & 0.0 & 200.0 & 120.0 & -0.001 \\
\hline Pugettia quadridens & 5.6 & 1.57 & 1.50 & 0.4 & 0.4 & 0.0 & 15.0 & 14.1 & 5.6 & 3.9 & 0.0 & 100.0 & 97.1 & 0.115 \\
\hline oetus & 5.6 & 4.97 & 6.99 & 4.4 & 3.3 & 0.0 & 110.0 & 89.3 & 11.1 & 7.7 & 0.0 & 200.0 & 194.3 & 0.535 \\
\hline apsus sanqui & 13.9 & 8.17 & 10.32 & 4.8 & 3.9 & 0.0 & 140.0 & 113.3 & 22.2 & 11.3 & 1.0 & 300.0 & 208.6 & -0.037 \\
\hline Pachycheles stevensii & 2.8 & 0.28 & 0.00 & 0 . & 0 & 0.0 & 1.0 & 1.0 & 2.8 & 2.8 & .0 & 100.0 & 100.0 & 0.411 \\
\hline hura japor & 8.3 & 0.26 & 0.00 & 0.0 & 0.0 & 0.0 & 0.1 & 0.1 & 11.1 & 6.6 & 0 & 200.0 & 142.9 & 0.254 \\
\hline Gnor & 8.3 & 2.27 & 2.58 & 0.6 & 0 & 0.0 & 20.0 & 18.1 & 11.1 & 6.6 & .0 & 0.0 & 142.9 & 0.384 \\
\hline a sp. & 2.8 & 0.09 & 0.00 & 0 & 0 & 0.0 & 0.1 & 0.1 & 2.8 & 2.8 & .0 & 100.0 & 100.0 & -0.083 \\
\hline Munnidae gen, sp. & 5.6 & 0.12 & 0.00 & 0 . & 0 & 0.0 & 0.1 & 0.1 & 2.8 & 2.8 & .0 & 100.0 & 100.0 & -0.083 \\
\hline Ianiropsis serrican & 2.8 & 0.09 & 0.00 & 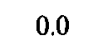 & & 0.0 & 0.1 & 0.1 & 2.8 & 2.8 & 0 & 100.0 & 100.0 & -0.081 \\
\hline iorata & 100.0 & 36.71 & 160.39 & 13.5 & & 0.0 & 180.0 & 76.7 & 1905.6 & 559.7 & 0.0 & 15000.0 & 5917.4 & -0.092 \\
\hline a cristibrac & 47.2 & 20.52 & 56.26 & & & 0.0 & 70.0 & 31.6 & 355.6 & 118.6 & 0.0 & 3500.0 & 1424.6 & 0.013 \\
\hline mutica & 11.1 & 1.92 & 1.58 & & & 0.0 & 65 & 4.5 & 8.3 & 4.7 & 0 & 100.0 & 94.3 & 0.218 \\
\hline lecta & 30.6 & 4.11 & 6.71 & & & 0.0 & 8.0 & 4.1 & 75.0 & 28.0 & .0 & 0.0 & 376.2 & 0.129 \\
\hline sp. & 44.4 & 7.11 & 14.41 & & & 0.0 & 10.0 & 6.0 & 188.9 & 64.3 & 0.0 & 2100.0 & 788.9 & 0.232 \\
\hline Con & 5.6 & 0.18 & 0.00 & 0 . & & 0.0 & 0 . & 0.1 & 5.6 & 3.9 & .0 & 100.0 & 97.1 & -0.058 \\
\hline & 2.8 & 0.09 & 0.00 & & 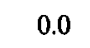 & 0.0 & 0.1 & 0.1 & 2.8 & .8 & 1.0 & 100.0 & 100.0 & -0.017 \\
\hline Is & 13.9 & 1.04 & 1.58 & & & 0.0 & 1.0 & 0.8 & 25.0 & 11.5 & 0.0 & 300.0 & 191.4 & 0.225 \\
\hline Metopelloides bar & 5.6 & 0.28 & 0.00 & 0.0 & & 0.0 & 0. & 0.3 & 11.1 & 8.7 & 0.0 & 300.0 & 245.7 & $7-0.084$ \\
\hline Metopelloides sch & 2.8 & 0.09 & 0.00 & 0.0 & 0.0 & 0.0 & 0.1 & 0.1 & 2.8 & 2.8 & 0.0 & 100.0 & 100.0 & 0.010 \\
\hline Pare & 2.8 & 0.15 & 0.00 & & O & 0.0 & 0.3 & 0.3 & 8.3 & 8.3 & 0.0 & 0.0 & 300.0 & -0.081 \\
\hline Parhy & 2.8 & 0.09 & 0.00 & & & 0.0 & 0.1 & 0.1 & 2.8 & 2.8 & 0.0 & 100.0 & 100.0 & -0.048 \\
\hline Pleus. & 19.4 & 1.04 & 1.67 & 0.1 & & 0.0 & 1.0 & 0.6 & 27.8 & 12.4 & 0.0 & 400.0 & 197.7 & $7-0.079$ \\
\hline & 11.1 & 0.35 & 0.00 & 0.0 & 0.0 & 0.0 & 0.1 & 0.1 & 16.7 & 10.2 & 0.0 & 300.0 & 222.9 & 9.220 \\
\hline Lecythorhyncus & 2.8 & 0.28 & 0.00 & & 0 & 0.0 & 1.0 & 1.0 & 2.8 & 2.8 & 0.0 & 100.0 & 100.0 & $0-0.021$ \\
\hline Epheria turrita & 13.9 & 4.44 & 12.00 & 1.4 & 1.1 & 0.0 & 40.0 & 31.8 & 102.8 & 63.2 & 0.0 & 2100.0 & 1398.2 & $2 \quad 0.406$ \\
\hline Littorina mandsc & 2.8 & 0.09 & 0.00 & 0.0 & 0.0 & 0.0 & 0.1 & 0.1 & 2.8 & 2.8 & 0.0 & 100.0 & 100.0 & $\begin{array}{ll}0 & 0.088\end{array}$ \\
\hline Alve & 11.1 & 0.35 & 0.00 & 0.0 & 0.0 & 0.0 & 0.1 & 0.1 & 11.1 & 5.3 & 0.0 & 0.0 & 91.4 & $4 \quad 0.563$ \\
\hline Anadara brought & 11.1 & 0.35 & 0.00 & 0.0 & 0. & 0. & 0 . & 0.1 & 11.1 & 6.6 & 0.0 & 200.0 & 142.9 & $\begin{array}{ll}9 & 0.329\end{array}$ \\
\hline Arca boucardi & 11.1 & 0.31 & 0.00 & 0.0 & 0.0 & 0.0 & 0.2 & 0.2 & 13.9 & 8.1 & 0.0 & 200.0 & 170.9 & $9 \quad 0.333$ \\
\hline Callista breviph & 22.2 & 0.82 & 0.00 & 0.0 & 0.0 & 0.0 & 0.3 & 0.1 & 33.3 & 12.6 & 0.0 & 300.0 & 171.4 & $4 \quad 0.237$ \\
\hline Callithaca adamsi & 36.1 & 3.14 & & 0.3 & 0.2 & 0.0 & 8.0 & 6.5 & 105.6 & 35.8 & 0.0 & 800.0 & 438.2 & $2 \quad 0.427$ \\
\hline Crenomytilus graya & 19.4 & 2.65 & 5.06 & 0.4 & 0.3 & 0.0 & 10.0 & 7.9 & 63.9 & 30.9 & 0.0 & 1000.0 & 538.0 & $0 \quad 0.406$ \\
\hline Crassostrea gigas & 11.1 & 10.39 & 14.67 & 9.7 & 6.2 & 0.0 & 180.0 & 144.3 & 22.2 & 12.0 & 0.0 & 300.0 & 234.3 & $3-0.004$ \\
\hline Entodesma naviculoides & 2.8 & 0.03 & 0.00 & 0.0 & 0.0 & 0.0 & 0.0 & 0.0 & 2.8 & 2.8 & 0.0 & 100.0 & 100.0 & $0-0.017$ \\
\hline Hiatella anctica & 44.4 & 4.70 & 9.65 & 0.5 & 0.3 & 0.0 & 10.0 & 5.7 & 186.1 & 47.8 & 0.0 & 1300.0 & 442.6 & $6-0.030$ \\
\hline Mizuhopecten yessoensis & 2.8 & 0.12 & & 0.0 & 0.0 & 0.0 & 0.2 & 0.2 & - & - & - & - & - & 0.022 \\
\hline
\end{tabular}


Table 2. Continued.

\begin{tabular}{|c|c|c|c|c|c|c|c|c|c|c|c|c|c|c|}
\hline \multirow{2}{*}{ pecies } & & \multirow{2}{*}{$\sqrt{F \times B}$} & \multirow{2}{*}{$\sqrt{A \times B}$} & \multicolumn{5}{|c|}{ Biomass, $\mathrm{g} / \mathrm{m}^{2}$} & \multicolumn{5}{|c|}{ Population density, ind $/ \mathrm{m}^{2}$} & \multirow{2}{*}{$R$} \\
\hline & & & & $\bar{X}$ & $S E$ & $M I N$ & $M A X$ & $I_{W B}$ & $\bar{X}$ & $S E$ & $M I N$ & $M A X$ & $I_{W}$ & \\
\hline 1 & 2 & 3 & 4 & 5 & 6 & 7 & 8 & 9 & 10 & 11 & 12 & 13 & 14 & 15 \\
\hline Modiolus sp. & 2.8 & 0.09 & 0.00 & 0.0 & 0.0 & 0.0 & 0.1 & 0.1 & 2.8 & 2.8 & 0.0 & 100.0 & 100.0 & -0.076 \\
\hline Musculista senhousia & 2.8 & 0.09 & 0.00 & 0.0 & 0.0 & 0.0 & 0.1 & 0.1 & 2.8 & 2.8 & 0.0 & 100.0 & $100.0-$ & -0.045 \\
\hline Mya pseudoarenaria & 2.8 & 0.09 & 0.00 & 0.0 & 0.0 & 0.0 & 0.1 & 0.1 & 11.1 & 7.7 & 0.0 & 200.0 & $194.3-$ & -0.021 \\
\hline Mya japonica & 25.0 & 0.83 & 0.00 & 0.0 & 0.0 & 0.0 & 0.2 & 0.1 & 41.7 & 15.1 & 0.0 & 300.0 & $197.1-$ & -0.004 \\
\hline Mya sp. juv. & 2.8 & 0.12 & 0.00 & 0.0 & 0.0 & 0.0 & 0.2 & 0.2 & 5.6 & 3.9 & 0.0 & 100.0 & 97.1 & 0.757 \\
\hline Mytilus trossulus & 66.7 & 372.04 & 1520.75 & 2076.2 & 965.7 & 0.0 & 28000.01 & 16171.2 & 1113.9 & 325.6 & 0.0 & 8200.0 & 3426.9 & 0.985 \\
\hline Ruditapes philippinarum & 22.2 & 1.11 & 1.82 & 0.1 & 0.0 & 0.0 & 1.0 & 0.6 & 33.3 & 12.0 & 0.0 & 300.0 & 154.3 & 0.337 \\
\hline Turtonia minuta & 11.1 & 0.35 & 0.00 & 0.0 & 0.0 & 0.0 & 0.1 & 0.1 & 16.7 & 10.2 & 0.0 & 300.0 & $222.9-$ & -0.050 \\
\hline Bugula pacifica & 33.3 & 8.28 & - & 2.1 & 0.8 & 0.0 & 20.0 & 10.5 & - & - & - & $\cdot$ & - & 0.065 \\
\hline Conopeum seurati & 8.3 & 0.96 & - & 0.1 & 0.1 & 0.0 & 2.0 & 1.4 & - & - & - & - & - & -0.021 \\
\hline Amphiodia fissa & 11.1 & 0.66 & 0.00 & 0.0 & 0.0 & 0.0 & 1.0 & 0.7 & 14.3 & 7.2 & 0.0 & 200.0 & 129.4 & 0.863 \\
\hline steria japon & 2.8 & 2.78 & 0.00 & 2.8 & 2.8 & 0.0 & 100.0 & 100.0 & 0.0 & 0.0 & 0.0 & 0.0 & 0.0 & 0.394 \\
\hline ava & 33.3 & 2.72 & 0.75 & 0.2 & 0.2 & 0.0 & 8.0 & 8.0 & 2.8 & 2.8 & 0.0 & 100.0 & 100.0 & 0.011 \\
\hline Total & & & 4403.74 & 2788.1 & 951.6 & 2.1 & 28015.6 & 1667.1 & 6955.6 & 1174.2 & 300.0 & 27600.0 & 7135.8 & \\
\hline \multicolumn{15}{|c|}{ Tunnel bottom } \\
\hline Obelia longissima & 50.0 & 5.10 & - & 0.5 & 0.3 & 0.0 & 2.0 & 1.4 & - & - & - & - & - & 0.766 \\
\hline Halosydna breviset & 10.0 & 1.00 & - & 0.1 & 0.1 & 0 . & 1.0 & 1.0 & - & - & - & - & - & 0.318 \\
\hline Hydmides ezoensi & 50.0 & 16.12 & 21.63 & 5.2 & 2.3 & 0.0 & 20.0 & 10.3 & 90.0 & 40.7 & 0.0 & 400.0 & 184.0 & -0.139 \\
\hline Potamilla sp. & 40.0 & 8.72 & 8.72 & 1.9 & 1.1 & 0.0 & 10.0 & 6.1 & 40.0 & 22.1 & 0.0 & 200.0 & 122.2 & -0.244 \\
\hline multignatha & 20.0 & 1.48 & 1.48 & 0.1 & 0.1 & 0. & 1.0 & 0.9 & 20.0 & 13.3 & 0.0 & 100.0 & 88.8 & -0.259 \\
\hline $\mathrm{Ner}$ & 10.0 & 1.00 & 1.00 & 0.1 & 0.1 & 0.0 & 1.0 & 1.0 & 10.0 & 10.0 & 0 & 100.0 & 100.0 & 0.338 \\
\hline llis orientalis & 10.0 & 0.32 & 0.32 & 0.0 & 0.0 & 0.0 & 0.1 & 0.1 & 10.0 & 10.0 & 0.0 & 100.0 & 100.0 & 0.318 \\
\hline Balanus rostratus & 40.0 & 38.68 & 98.61 & 37.4 & 21.0 & 0.0 & 180.0 & 117.3 & 260.0 & 155.8 & 0.0 & 1500.0 & 933.3 & 0.198 \\
\hline Balanus improvisus & 20.0 & 9.80 & 16.97 & 4.8 & 4.0 & 0.0 & 40.0 & 33.2 & 60.0 & 42.7 & 0.0 & 400.0 & 303.7 & -0.101 \\
\hline quadridens & 20.0 & 5.66 & 5.66 & 1.6 & 1.5 & 0.0 & 15.0 & 13.9 & 20.0 & 13.3 & 0.0 & 100.0 & 88.9 & -0.034 \\
\hline Can & 20.0 & 17.89 & 25.30 & 16.0 & 11.6 & 0.0 & 110.0 & 83.6 & 40.0 & 26.7 & 0.0 & 200.0 & 177.8 & 0.449 \\
\hline $\mathrm{Hem}$ & 30.0 & 21.42 & 27.66 & 15.3 & 13.9 & 0 & 140.0 & 126.0 & 50.0 & 34.2 & 0 & 300.0 & 233.3 & -0.228 \\
\hline Pact & 10.0 & 1.00 & 1.00 & 0.1 & 0.1 & 0.0 & 1.0 & 1.0 & 10.0 & 10.0 & 0 & 100.0 & 100.0 & 0.338 \\
\hline Para & 10.0 & 0.32 & 0.32 & 0.0 & 0.0 & 0.0 & 0.1 & 0.1 & 10.0 & 10.0 & 1.0 & 100.0 & 100.0 & 0.318 \\
\hline Gnor & 20.0 & 6.36 & 7.78 & 2.0 & 2.0 & 0.0 & 20.0 & 19.8 & 30.0 & 21.3 & 1.0 & 200.0 & 151.9 & 0.316 \\
\hline Jassa marmorata & 100.0 & 47.98 & 189.40 & 23.0 & 17.6 & 0.1 & 180.0 & 134.0 & 1560.0 & 617.7 & 100.0 & 6500.0 & 2446.2 & -0.267 \\
\hline Cap & 60.0 & 26.04 & 53.15 & 11.3 & 6.8 & 0.0 & 70,0 & 40.3 & 250.0 & 134.4 & 0.0 & 1400.0 & 722.2 & -0.151 \\
\hline tica & 10.0 & 2.00 & 2.00 & & 0.4 & 0.0 & 40 & 4.0 & 10.0 & 10.0 & 0.0 & 100.0 & 100.0 & 0.338 \\
\hline neglecta & 50.0 & 5.66 & 7.59 & 0 & 0.4 & 0.0 & 4.0 & 4 & 90.0 & 37.9 & .0 & 300.0 & 159.3 & 0.210 \\
\hline Cap & 60.0 & 10.45 & 18.60 & 1.8 & 1.1 & 0.0 & 10.0 & 6.4 & 190.0 & 80.9 & 0 & 700.0 & 344.4 & 0.140 \\
\hline Coro & 10.0 & 0.32 & 0.32 & 0.0 & 0.0 & 0.0 & 0.1 & 0.1 & 10.0 & 10.0 & 0.0 & 100.0 & 100.0 & -0.235 \\
\hline rus sp. & 30.0 & 2.68 & 3.46 & 0. & 0.1 & 0.0 & 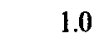 & 0.7 & 50.0 & 26.9 & 0.0 & 200.0 & 144.4 & 0.000 \\
\hline lloides barn & 10.0 & 0.32 & 0.32 & 0.0 & 0.0 & 0.0 & 0.1 & 0.1 & 10.0 & 10.0 & 0.0 & 100.0 & 100.0 & -0.191 \\
\hline Parh & 10.0 & 0.32 & 0.32 & 0.0 & 0.0 & 0.0 & 0.1 & 0.1 & 10.0 & 10.0 & 0.0 & 100.0 & 100.0 & -0.222 \\
\hline Plen & 30.0 & 1.34 & 1.34 & 0. & 0.0 & 0.0 & 0.4 & 0.3 & 30.0 & 15.3 & 0.0 & 100.0 & 77.8 & -0.181 \\
\hline Prostenothoe sextonae & 10.0 & 0.32 & 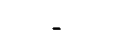 & 0 & 0 & 0.0 & 0.1 & 0.1 & - & - & - & - & - & 0.338 \\
\hline Epheria turrita & 50.0 & 15.98 & 43.48 & 5.1 & 3.9 & 0.0 & 40.0 & 30.2 & 370.0 & 211.9 & 0.0 & 2100.0 & 1213.5 & 0.296 \\
\hline Littorina mandsct & 10.0 & 0.32 & 0.32 & 0.0 & 0.0 & 0.0 & 0.1 & 0.1 & 10.0 & 10.0 & 0.0 & 100.0 & 100.0 & -0.056 \\
\hline Alvenius ojamus & 30.0 & 0.95 & 0.95 & 0.0 & 0.0 & 0.0 & 0.1 & 0.1 & 30.0 & 15.3 & 0.0 & 100.0 & 77.8 & 0.553 \\
\hline Anadara hroughtoni & 30.0 & 0.95 & 1.10 & 0.0 & 0.0 & 0.0 & 0.1 & 0.1 & 40.0 & 22.1 & 0.0 & 200.0 & 122.2 & 0.199 \\
\hline Arca boucardi & 20.0 & 0.77 & 1.10 & 0.0 & 0.0 & 0.0 & 0.2 & 0.2 & 40.0 & 26.7 & 0.0 & 200.0 & 177.8 & 0.194 \\
\hline Callista breviphonata & 30.0 & 1.22 & 1.41 & 0.1 & 0.0 & 0.0 & 0.3 & 0.2 & 40.0 & 26.7 & 0.0 & 200.0 & 177.8 & 0.165 \\
\hline Callithaca adamsi & 40.0 & 5.83 & 11.66 & 0.9 & 0.8 & 0.0 & 8.0 & 7.4 & 160.0 & 93.3 & 0.0 & 800.0 & 544.4 & 0.349 \\
\hline Crenomytilus graya & 50.0 & 7.97 & 15.53 & 1.3 & 1.0 & 0.0 & 10.0 & 7.7 & 190.0 & 101.6 & 0.0 & 1000.0 & 543.3 & 0.298 \\
\hline Crassostrea gtg(a) & 10.0 & 3.16 & 3.16 & 1.0 & 1.0 & 0.0 & 10.0 & 10.0 & 10.0 & 10.0 & 0.0 & 100.0 & 100.0 & -0.235 \\
\hline Hiatella arctica & 40.0 & 2.28 & 3.42 & 0.1 & 0.1 & 0.0 & 1.0 & 0.7 & 90.0 & 37.9 & 0.0 & 300.0 & 159.3 & -0.257 \\
\hline
\end{tabular}


Table 2. Continued.

\begin{tabular}{|c|c|c|c|c|c|c|c|c|c|c|c|c|c|c|}
\hline \multirow{2}{*}{ Species } & \multirow{2}{*}{$\mathbf{F}$} & \multirow{2}{*}{$\sqrt{F \times B}$} & \multirow{2}{*}{$\sqrt{A \times B}$} & \multicolumn{5}{|c|}{ Biomass, $g / m^{2}$} & \multicolumn{5}{|c|}{ Population density, ind $/ \mathrm{m}^{2}$} & \multirow{2}{*}{$R$} \\
\hline & & & & $\bar{X}$ & $S E$ & MIN & $M A X$ & $I_{H B}$ & $\bar{x}$ & $S E$ & $M I N$ & $M A X$ & $I_{W}$ & \\
\hline 1 & 2 & 3 & 4 & 5 & 6 & 7 & 8 & 9 & 10 & 11 & 12 & 13 & 14 & 15 \\
\hline Todiolus sp. & 10.0 & 0.32 & 0.32 & 0.0 & 0.0 & 0.0 & 0.1 & 0.1 & 10.0 & 10.0 & 0.0 & 100.0 & 100.0 & -0.256 \\
\hline Mya japonica & 10.0 & 0.32 & 0.00 & 0.0 & 0.0 & 0.0 & 0.1 & 0.1 & 0.0 & 0.0 & 0.0 & 0.0 & 100.0 & -0.056 \\
\hline Mya sp. juv. & 10.0 & 0.45 & 0.45 & 0.0 & 0.0 & 0.0 & 0.2 & 0.2 & 10.0 & 10.0 & 0.0 & 100.0 & 100.0 & 0.762 \\
\hline Mytilus trossulus & 100.0 & 842.91 & 4539.22 & 7105.0 & 3024.0 & 180.02 & 28000.01 & 12870.8 & 2900.0 & 950.7 & 400.0 & 8200.0 & 3116.5 & 1.000 \\
\hline Ruditapes philippi & 50.0 & 2.83 & 3.10 & 0.2 & 0.1 & 0.0 & 1.0 & 0.6 & 60.0 & 26.7 & 0.0 & 200.0 & 118.5 & 0.175 \\
\hline Turtonia minuta & 40.0 & 1.26 & 1.55 & 0.0 & 0.0 & 0.0 & 0.1 & 0.1 & 60.0 & 34.0 & 0.0 & 300.0 & 192.6 & -0.472 \\
\hline Bugula pacifica & 10.0 & 2.24 & - & 0.5 & 0.5 & 0.0 & 5.0 & 5.0 & - & - & - & - & - & 0.338 \\
\hline Amphiodia fissa & 30.0 & 1.97 & 2.28 & 0.1 & 0.1 & 0.0 & 1.0 & 0.8 & 40.0 & 22.1 & 0.0 & 200.0 & 122.2 & 0.870 \\
\hline Aphelasteriajapo & 10.0 & 10.00 & - & 10.0 & 10.0 & 0.0 & 100.0 & 100.0 & - & - & - & - & - & 0.318 \\
\hline \multicolumn{15}{|c|}{ Tunnel walls } \\
\hline Obelia longissima & 73.3 & 33.27 & - & 15.1 & 13.9 & 0.0 & 210.0 & 192.8 & - & - & - & - & - & 0.094 \\
\hline Hydr & 86.7 & 21.26 & 25.01 & 5.2 & 1.5 & 0.0 & 15.0 & 6.4 & 120.1 & 26.2 & 0.0 & 300.0 & 85.5 & 0.324 \\
\hline Potamilla sp. & 93.3 & 31.08 & 46.99 & 10.4 & 2.7 & 0.0 & 30.0 & 10.3 & 213.3 & 33.6 & 100.0 & 500.0 & 79.5 & 0.615 \\
\hline Nereis multignatha & 20.0 & 4.16 & 4.82 & 0.9 & 0.7 & 0.0 & 10.0 & 7.7 & 26.7 & 15.3 & 0.0 & 200.0 & 132.1 & 0.212 \\
\hline Nere & 6.7 & 0.67 & 0.68 & 0.1 & 0.1 & 0.0 & 1.0 & 1.0 & 6.7 & 6.7 & 0.0 & 100.0 & 100.0 & 0.238 \\
\hline Nereis sp. & 20.0 & 1.71 & 2.00 & 0.2 & 0.1 & 0.0 & 2.0 & 1.8 & 26.7 & 20.6 & 0.0 & 300.0 & 239.3 & 0.386 \\
\hline Balanus rostratus & 93.3 & 370.63 & 2624.50 & 1471.8 & 238.5 & 0.0 & 2650.0 & 579.7 & 4680.0 & 957.7 & 0.0 & 14000.0 & 2939.6 & 0.960 \\
\hline Balanus improvisus & 73.3 & 46.85 & 103.80 & 29.9 & 12.8 & 0.0 & 160.0 & 82.5 & 360.0 & 66.0 & 0.0 & 800.0 & 181.8 & -0.223 \\
\hline Balanus crenatus & 13.3 & 12.65 & 15.49 & 12.0 & 10.7 & 0.0 & 160.0 & 141.9 & 20.0 & 14.5 & 0.0 & 200.0 & 157.1 & 0.320 \\
\hline Hemigrapsus sang & 13.3 & 4.22 & 5.16 & 1.3 & 0.9 & 0.0 & 10.0 & 9.3 & 20.0 & 14.5 & 0.0 & 200.0 & 157.1 & 0.524 \\
\hline Paranthura japonica & 13.3 & 0.42 & 0.45 & 0.0 & 0.0 & 0.0 & 0.1 & 0.1 & 20.0 & 14.5 & 0.0 & 200.0 & 157.1 & 0.524 \\
\hline Mun & 6.7 & 0.21 & 0.26 & 0.0 & 0.0 & 0.0 & 0.1 & 0.1 & 6.7 & 6.7 & 0.0 & 100.0 & 100.0 & -0.439 \\
\hline Jasso & 100.0 & 30.43 & 134.95 & 9.3 & 5.4 & 0.1 & 82.0 & 47.9 & 1960.0 & 893.4 & 100.0 & 12000.0 & 6107.7 & 0.315 \\
\hline Caprella cristibrachium & 73.3 & 28.06 & 69.74 & 10 & 4.0 & 0.0 & 55.0 & 22.7 & 453.3 & 149.6 & 0.0 & 1700.0 & 740.1 & 0.380 \\
\hline tica & 13.3 & 2.67 & 2.66 & 0.5 & 0.4 & 0.0 & 6.0 & 4.8 & 13.3 & 9.1 & 0.0 & 100.0 & 92.9 & 0.229 \\
\hline Cap & 33.3 & 5.03 & - & 0.8 & 0.5 & 0.0 & 8.0 & 5.7 & 100.0 & 60.9 & 0.0 & 900.0 & 557.1 & 0.126 \\
\hline Cap & 33.3 & 7.01 & 19.80 & 1.5 & 0.7 & 0 & 7.0 & 5.6 & 266.7 & 142.7 & 0.0 & 2100.0 & 1144.6 & 0.461 \\
\hline um acher & 6.7 & 0.21 & 0.26 & 0.0 & 0.0 & & 0 & 0 & 6.7 & 6.7 & 0.0 & 100.0 & 0 & 0.049 \\
\hline & 6.7 & 0.21 & 0.26 & 0.0 & 0.0 & 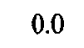 & 0.1 & 0.1 & 6.7 & 6.7 & 0.0 & 100.0 & 100.0 & 0.090 \\
\hline Ishyn & 13.3 & 0.60 & 0.89 & 0.0 & 0.0 & 0.0 & 0.3 & 0.2 & 26.7 & 20.6 & 0.0 & 300.0 & 239.3 & 0.400 \\
\hline Meto & 6 & 0.21 & 0.26 & 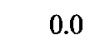 & 0.0 & 0. & 0 & 0 & 6.7 & 6.7 & 0.0 & 100.0 & 100.0 & 0.308 \\
\hline Pleu & 6.7 & 0.30 & 0.26 & 0.0 & 0.0 & 0.0 & 0.2 & 0.2 & 6.7 & 6.7 & 0.0 & 100.0 & 100.0 & -0.136 \\
\hline Pros & 20.0 & 0.63 & 0.89 & 0.0 & 0.0 & 0.0 & 0.1 & 0.1 & 40.0 & 23.5 & 0.0 & 300.0 & 207.1 & 0.477 \\
\hline Lecy & 6.7 & 0.67 & 0.68 & 0 . & 0.1 & 0 & 1. & 1. & 6.7 & 6.7 & 0.0 & 100.0 & 100.0 & 0.057 \\
\hline Alvenius ojanus & 6.7 & 0.21 & 0.26 & 0.0 & 0.0 & 0 & 0 & 0. & 6.7 & 6.7 & 0.0 & 100.0 & 100.0 & 0.090 \\
\hline Anadara brought & 6.7 & 0.21 & 0.00 & 0.0 & 0.0 & 0.0 & 0.1 & 0.1 & 0.0 & 0.0 & 0.0 & 0.0 & 100.0 & 0.049 \\
\hline Arca boucandi & 6.7 & 0.07 & 0.00 & 0.0 & 0.0 & 0.0 & 0.0 & 0.0 & 6.7 & 6.7 & 0.0 & 100.0 & 100.0 & -0.136 \\
\hline Callista breviphona & 40.0 & 1.26 & 1.46 & 0.0 & 0.0 & 0.0 & 0 . & 0.1 & 53.3 & 23.6 & 0.0 & 300.0 & 157.1 & -0.107 \\
\hline Callithaca adamsi & 46.7 & 2.02 & 3.63 & 0.1 & 0.0 & 0.0 & 0.3 & 0.1 & 146.7 & 56.0 & 0.0 & 700.0 & 320.1 & 0.614 \\
\hline Creno & 13.3 & 0.52 & 0.73 & 0.0 & 0.0 & 0.0 & 0.2 & 0.2 & 26.7 & 15.3 & 0.0 & 200.0 & 132.1 & 0.120 \\
\hline Crass & 20.0 & 21.29 & 32.53 & 22.7 & 14.6 & 0.0 & 180.0 & 140.8 & 46.7 & 27.4 & 0.0 & 300.0 & 240.8 & 0.338 \\
\hline Entodesma navicul & 6.7 & 0.07 & 0.00 & 0.0 & 0.0 & 0.0 & 0.0 & 0.0 & 6.7 & 6.7 & 0.0 & 100.0 & 100.0 & 0.089 \\
\hline Hiatella arctica & 80.0 & 9.38 & 20.45 & 1.1 & 0.7 & 0.0 & 10.0 & 5.8 & 380.0 & 91.1 & 0.0 & 1300.0 & 327.8 & 0.169 \\
\hline Mizuhopecten yessoens & 6.7 & 0.30 & 0.00 & 0.0 & 0.0 & 0.0 & 0.2 & 0.2 & 0.0 & 0.0 & 0.0 & 0.0 & 100.0 & 0.401 \\
\hline Musculista senhousia & 6.7 & 0.21 & 0.26 & 0.0 & 0.0 & 0.0 & 0.1 & 0.1 & 6.7 & 6.7 & 0.0 & 100.0 & 100.0 & -0.136 \\
\hline Mya pseudoarenaria & 6.7 & 0.21 & 0.52 & 0.0 & 0.0 & 0.0 & 0.1 & 0.1 & 26.7 & 18.2 & 0.0 & 200.0 & 185.7 & 0.057 \\
\hline Mya japonica & 46.7 & 1.67 & - & 0.1 & 0.0 & 0.0 & 0.2 & 0.1 & 100.0 & 30.9 & 0.0 & 300.0 & 142.9 & 0.428 \\
\hline Mytilus trossulus & 100.0 & 156.89 & 26.77 & 46.1 & 85.4 & 1.0 & 1320.0 & 444.3 & 740.0 & 148.3 & 100.0 & 1800.0 & 445.6 & 0.634 \\
\hline Ruditapes philippi & 13.4 & 0.61 & 1.10 & 0.0 & 0.0 & 0.0 & 0.3 & 0.2 & 40.0 & 21.4 & 0.0 & 300.0 & 171.4 & 0.376 \\
\hline Bugula pacifica & 73.3 & 18.38 & - & 4.6 & 1.6 & 0.0 & 20.0 & 8.7 & - & - & - & - & - & 0.559 \\
\hline
\end{tabular}


Table 2. Continued.

\begin{tabular}{|c|c|c|c|c|c|c|c|c|c|c|c|c|c|c|}
\hline \multirow{2}{*}{ Species } & \multirow{2}{*}{$\mathbf{F}$} & \multirow{2}{*}{$\sqrt{F \times B}$} & \multirow{2}{*}{$\sqrt{A \times B}$} & \multicolumn{5}{|c|}{ Biomass, $\mathbf{g} / \mathrm{m}^{2}$} & \multicolumn{5}{|c|}{ Population density, ind $/ \mathrm{m}^{2}$} & \multirow{2}{*}{$\boldsymbol{R}$} \\
\hline & & & & $\bar{X}$ & $S E$ & $M I N$ & $M A X$ & $I_{W B}$ & $\bar{X}$ & $S E$ & $M I N$ & $M A X$ & $I_{w}$ & \\
\hline 1 & 2 & 3 & 4 & 5 & 6 & 7 & 8 & 9 & 10 & 11 & 12 & 13 & 14 & 15 \\
\hline Conopeum seurati & 20.0 & 2.31 & - & 0.3 & 0.2 & 0.0 & 2.0 & 1.3 & - & - & - & - & - & 0.220 \\
\hline Amphiodia fissa & 6.7 & 0.21 & 0.26 & 0.0 & 0.0 & 0.0 & 0.1 & 0.1 & 6.7 & 6.7 & 0.0 & 100.0 & 100.0 & 0.089 \\
\hline Styela clava & 6.7 & 1.89 & - & 0.5 & 0.5 & 0.0 & 8.0 & 8.0 & - & - & - & - & - & 0.313 \\
\hline \multicolumn{15}{|c|}{ Tunnel vault } \\
\hline Obelia longissima & 63.6 & 11.93 & - & 2.2 & 1.3 & 0.0 & 15.0 & 8.7 & - & - & - & - & - & 0.345 \\
\hline Hydroides ezoensis & 9.1 & 0.91 & 0.90 & 0.1 & 0.1 & 0.0 & 1.0 & 1.0 & 9.1 & 9.1 & 0.0 & 100.0 & 100.0 & 0.947 \\
\hline Potamilla sp. & 9.1 & 0.91 & 1.28 & 0.1 & 0.1 & 0.0 & 1.0 & 1.0 & 18.2 & 18.2 & 0.0 & 200.0 & 200.0 & 0.947 \\
\hline Dexiospira alveolata & 9.1 & 0.91 & 0.90 & 0.1 & 0.1 & 0.0 & 1.0 & 1.0 & 9.1 & 9.1 & 0.0 & 100.0 & 100.0 & 0.947 \\
\hline Balamus rostratus & 27.3 & 2.77 & 2.76 & 0.3 & 0.2 & 0.0 & 2.0 & 1.5 & 27.3 & 14.1 & 0.0 & 100.0 & 80.0 & -0.174 \\
\hline Balanus improvisus & 18.2 & 2.23 & 2.71 & 0.3 & 0.2 & 0.0 & 2.0 & 1.5 & 27.3 & 19.5 & 0.0 & 200.0 & 153.3 & 0.051 \\
\hline Balanus crenatus & 27.3 & 6.49 & 6.50 & 1.6 & 0.9 & 0.0 & 8.0 & 5.4 & 27.3 & 14.1 & 0.0 & 100.0 & 80.0 & 0.824 \\
\hline Gnorimosphaeroma ovatum & 9.1 & 1.29 & 1.28 & 0.2 & 0.2 & 0.0 & 2.0 & 2.0 & 9.1 & 9.1 & 0.0 & 100.0 & 100.0 & 0.106 \\
\hline Limnoria sp. & 9.1 & 0.29 & 0.30 & 0.0 & 0.0 & 0.0 & 0.1 & 0.1 & 9.1 & 9.1 & 0.0 & 100.0 & 100.0 & 0.106 \\
\hline Ianimpsis serricandis & 9.1 & 0.29 & 0.30 & 0.0 & 0.0 & 0.0 & 0.1 & 0.1 & 9.1 & 9.1 & 0.0 & 100.0 & 100.0 & 0.947 \\
\hline Jassa marmorata & 100.0 & 32.47 & 150.45 & 10.6 & 2.9 & 2.0 & 35.0 & 8.9 & 2145.5 & 1317.2 & 200.0 & 15000.0 & 8895.4 & 0.938 \\
\hline Caprella cristibrachium & 9.1 & 6.23 & 36.86 & 4.3 & 4.3 & 0.0 & 47.0 & 47.0 & 318.2 & 318.2 & 0.0 & 3500.0 & 3500.0 & 0.947 \\
\hline Caprella neglecta & 18.2 & 1.86 & 2.28 & 0.2 & 0.2 & 0.0 & 2.0 & 1.9 & 27.3 & 19.5 & 0.0 & 200.0 & 153.3 & 0.945 \\
\hline Caprella sp. & 45.5 & 1.57 & 2.02 & 0.1 & 0.0 & 0.0 & 0.2 & 0.1 & 81.8 & 35.2 & 0.0 & 400.0 & 166.7 & 0.679 \\
\hline Metopelloides barnardi & 9.1 & 0.57 & 1.04 & 0.0 & 0.0 & 0.0 & 0.4 & 0.4 & 27.3 & 27.3 & 0.0 & 300.0 & 300.0 & 0.947 \\
\hline Pareurystheus gurjanovae & 9.1 & 0.50 & 0.90 & 0.0 & 0.0 & 0.0 & 0.3 & 0.3 & 27.3 & 27.3 & 0.0 & 300.0 & 300.0 & 0.947 \\
\hline Pleusymtes sp. & 27.3 & 1.72 & 2.45 & 0.1 & 0.1 & 0.0 & 1.0 & 0.8 & 54.6 & 36.6 & 0.0 & 400.0 & 270.0 & 0.922 \\
\hline Hiatella anctica & 9.1 & 0.29 & 0.30 & 0.0 & 0.0 & 0.0 & 0.1 & 0.1 & 9.1 & 9.1 & 0.0 & 100.0 & 100.0 & 0.947 \\
\hline
\end{tabular}

Note. F - frequency, B - biomass, A - population density, $\bar{X}$, MIN and MAX - average, minimal and maximal values, SE - standard error, $I_{W}$ - dispersion index, $I_{W B}$ - dispersion index for biomass, $R$ - correlation coefficient of the biomass of separate species and total biomass; statistically significant $(\mathrm{p}<0.05)$ correlation coefficients and $I_{W}$ indices for statistically significant contagious animal distribution are in bold.
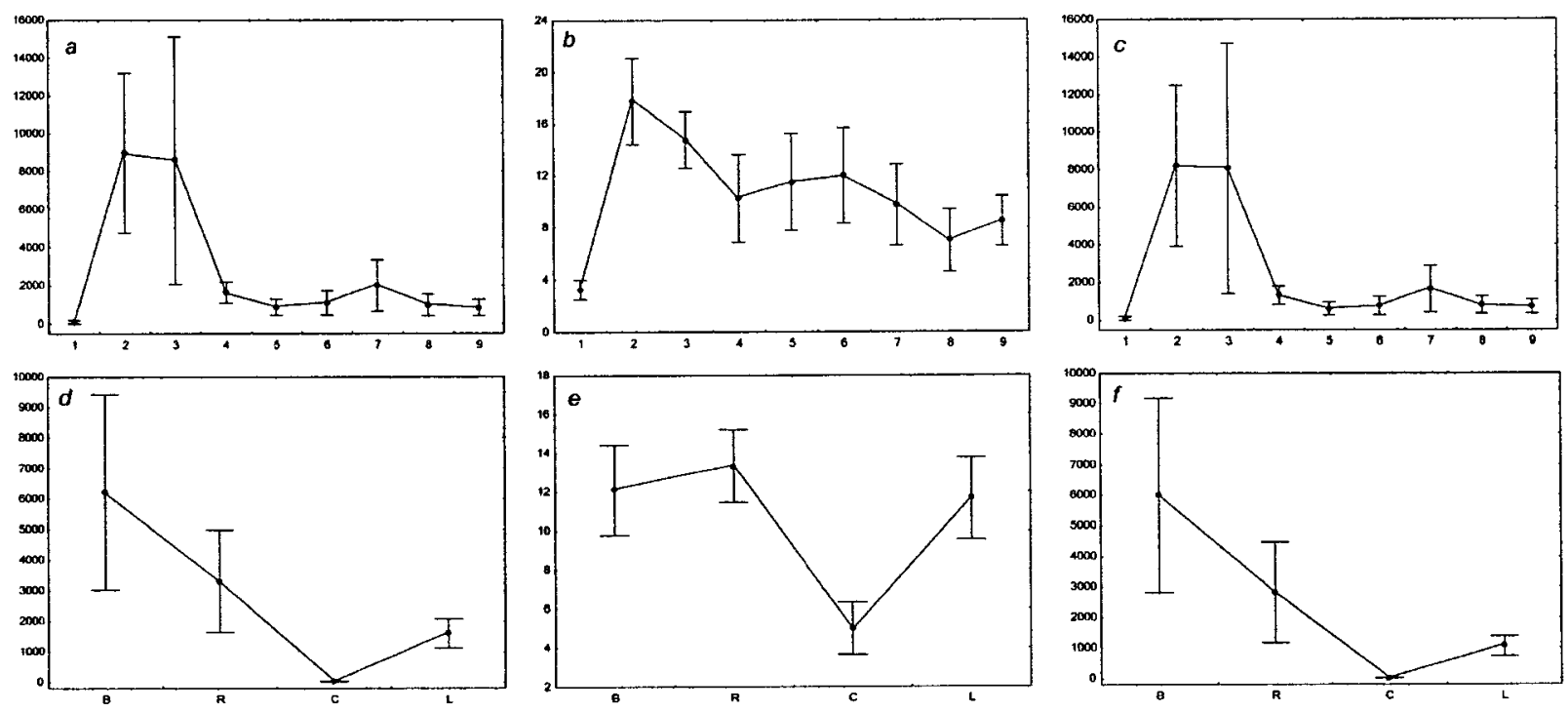

Fig. 2. Distribution of total biomass $(a, d)$, number of species $(b, e)$, and values of aggregation index $(c, f)$ of the total biomass of fouling organisms along the length $(a-c)$ and cross-sectional perimeter $(d-f)$ of the water intake tunnel of the cooling system of the Vladivostok Heat and Power Plant. Vertical lines - standard error; abscissa axis: $1-9$ - numbers of sections, $B$ - bottom, $C$ - vault, $L$ and $R$ - left and right walls of the tunnel, respectively. 

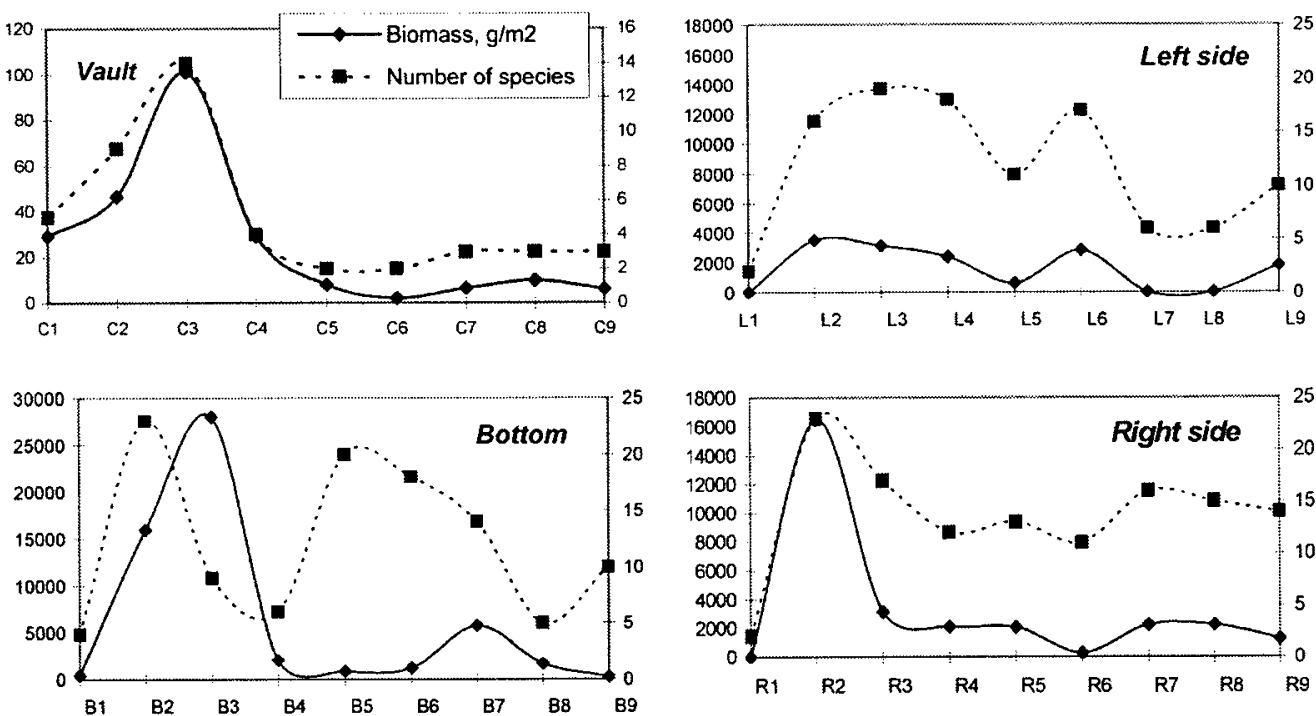

Fig. 3. Distribution of total biomass and species number of fouling organisms found on the vault, bottom, and walls of the water intake tunnel of the cooling system of Vladivostok Heat and Power Plant.

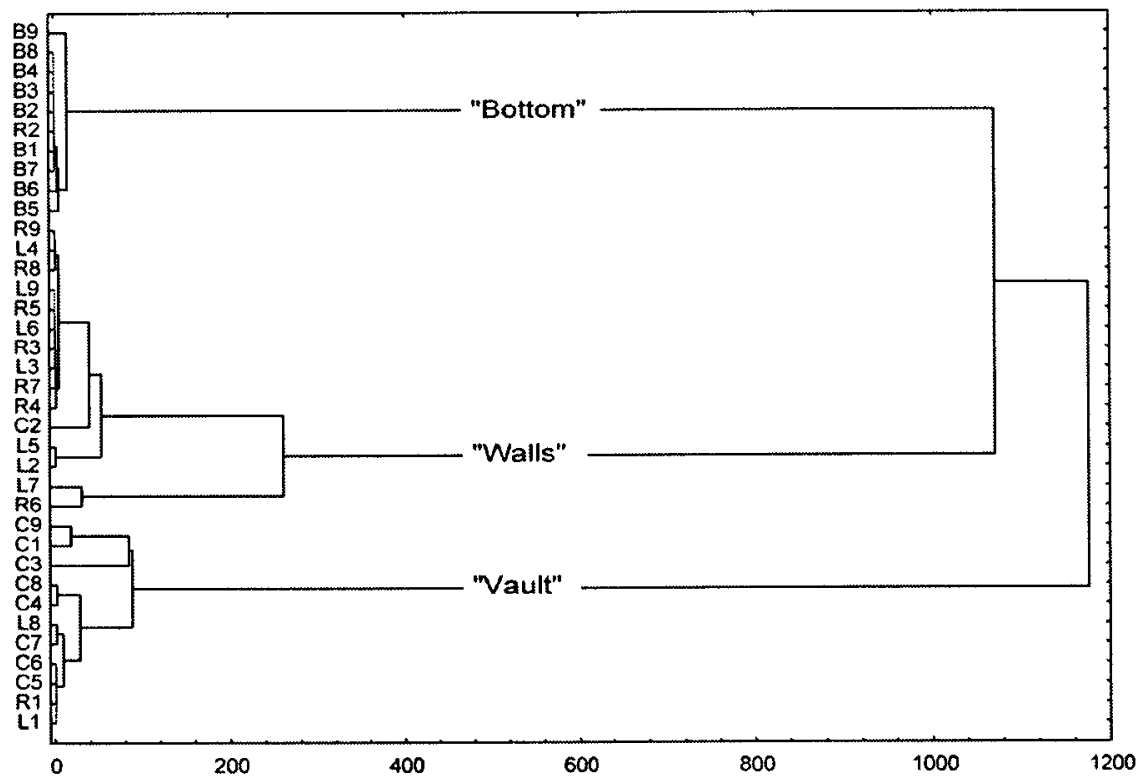

Fig. 4. Dendrogram of similarity for stations according to the preliminarily standardized biomass values of separate animal species obtained by Ward's method (metrics - squared Euclidean distance). Abscissa axis - within group sum of squared deviations.

tunnel (section 3), and the greatest species number also on the bottom and on the right wall (section 2). On the bottom of the tunnel, the maximums for total biomass and number of fouler species do not coincide with each other. The greatest biomass was observed immediately after the first turn and at the second turn (sections 3 and 7), with the second maximum approximately 5 times smaller than the first, and the greatest species number was found at the first turn (section 2) and in the middle of the tunnel (section 5). At the next two points, the species number was slightly reduced. On the vault and walls, surface maximums of biomass and species number on the whole coincide and fall on sections 2-4 and 6 on the left wall, section 2 on the right wall and section 3 on the vault of the tunnel. At that, the distribution of species number along the right wall was appreciably more uniform than along 
all the other "topological" subdivisions.

Distribution of all solitary animals without exception, both attached and motile, has a statistically significant aggregated (contagious) character, and the biomasses of individual animal species in most cases weakly correlates with total biomass (Table 2). At that, the degree of total biomass aggregation changes in accordance with total biomass variations: its maximums fall on sections 2 and 3 , and on the tunnel bottom, and $I_{w b}$ on the right wall is on the whole higher than that on the left (Fig. 2).

Most of the found animal species occur occasionally (Table 2), that is why not all species were used for classification of sampling points, but only those, which were met in $30 \%$ or more of cases (not less than in 11 samples from 36). Sample classification using the cluster analysis method showed the presence of three distinct groups, rather homogenous in terms of "topological" composition (Fig. 4). The first group includes samples collected from the bottom (sample R2 is an exception); the second groupsamples from the tunnel walls (except C2); and the third group-samples from the tunnel vault (with the exception of R1, L1 and L8). According to this kind of sorting, three groups of fouling animals were distinguished: 1-those found on the tunnel bottom, 2-those found on the tunnel walls, and 3-those found on the tunnel vault (Table 2).

The bottom group should be designated as being part of the Mytulis trossulus community according to biomass, frequency and $\sqrt{F \times B}$ index values (Table 2). This species dominates here absolutely. Subdominant species are $J$. marmorata and B. rostratus. The first of these, as well as $M$. trossulus, has $100 \%$ frequency, the second occupies the $2^{\text {nd }}$ place after mussels by biomass, and according to the $\sqrt{F \times B}$ index, it is the third after mussels and amphipods. At the same time, $B$. rostratus frequency is not great and comprises only $40 \%$. $M$. trossulus also have maximal values of $\sqrt{A \times B}$ index, 4539.2, and its values for amphipods and barnacles are noticeably smaller, 189.4 and 98.6 , correspondingly.

$B$. rostratus dominates according to biomass and the $\sqrt{F \times B}$ index in the group from the tunnel walls, and $M$. trossulus occupies the $2^{\text {nd }}$ place on the basis of these parameters (Table 2). In terms of frequency, B. rostratus shares $2^{\text {nd }}$ place with sedentary polychaete Potamilla $s p$. (93.7\%), while first place is occupied by $M$. trossulus and $J$. marmorata $(100 \%)$. B. rostratus was not found in one sample (L7), and this is the reason for the low threshold of this sample inclusion in group 2 (Fig. 4). The other acorn barnacle $B$. improvisus dominated here. Taking into account these facts, as well as the $\sqrt{A \times B}$ index values (2624.5,
426.8 and 135.0 for barnacle, mussel and amphipods, correspondingly), this group should be designated as the Balanus rostratus community. It is necessary to note an appreciably smaller significance of $J$. marmorata according to biomass in this community: except for mussels, it is surpassed by acom barnacle $B$. improvisus, oyster Crassostrea gigas, hydroid $O$. longissima, polychaete Potamilla sp. and other amphipod Caprella cristibrachium. All average numerical characteristics of $B$. rostratus were maximal for precisely this group.

In the group from the tunnel vault, $J$. marmorata is a dominant species by all parameters (Table 2). In this case, it reaches the maximal average population density among all groups at this point-2145.5 ind. $/ \mathrm{m}^{2}$. At the same time, the values of its biomass and $\sqrt{F \times B}$ index in this group are noticeably smaller than in bottom community, and approximately the same (slightly greater) as that of the group found at the tunnel walls. Hydroid $O$. longissima is the second species according to importance in the tunnel vault community (Jassa marmorata community). It is necessary to stress, that in contrast to groups found at the bottom and along the walls, in the case of the vault group, the biomass of most species closely correlates with the total biomass.

Distribution of most fouling species is rather specific (1-r tends to 1, Fig. 5). The greatest values of 1-r are usually observed for species with extremely low frequencies. Exclusion of these species from further analysis is advisable in revealing the categories for precise matching of dominant and subdominant species distribution, and in that way to investigate more thoroughly the functional relations of the most frequently occurring members of these communities.

Taking into account the evident "topological" unity of the obtained groups, the biomass data of species found in samples, collected from the tunnel bottom, vault and walls were included separately in the procedure of factor analysis. Such fragmentation of the total data array limits the number of species used in calculations to 9 for the bottom and vault, and 18 for the tunnel walls because the number of variables-biomasses of individual species-should not exceed the number of measurements. As a result of this limitation in the analysis (for bottom and vault) species, the smallest average arithmetic sum of the biomass and frequency scores were used (the greatest biomass and frequency is equal to 1 , second place-2, etc.).

In a number of cases purely "topological" final division of data was also based on the results of preliminary analysis of the distinguished clusters. For example, in the bottom group (being intermediate ones, the obtained results are 


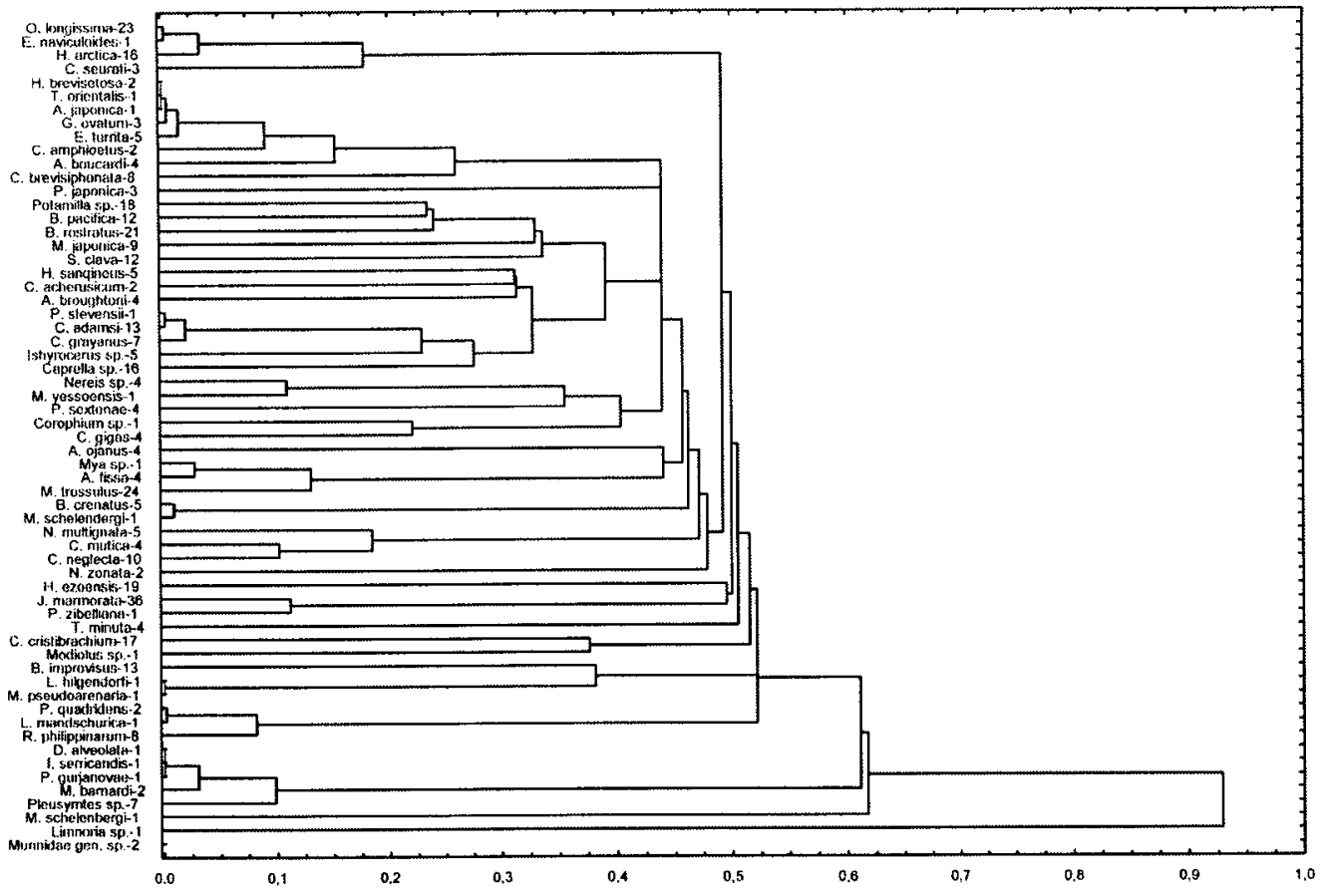

Fig. 5. Dendrogram of similarity for separate macrozoobenthos species according to distribution in the water intake tunnel of the cooling system of Vladivostok Heat and Power Plant, obtained by the method of single linkage (metrics - Pearson's correlation coefficient, $r$ ). Abscissa axis - I-r. The number of samples, where a species was found, is presented near a species' name.

not show in detail) sample $\mathrm{R} 2$ showed the maximum of the factor, which includes $B$. rostratus and Crenomytilus grayanus, on the background of mean value of the factor combining Epheria turrita, M. trossulus, and $O$. longissima. Therefore, this point can be referred to the area of ecotone between the communities of $M$. trossulus (tunnel bottom) and $B$. rostratus (tunnel walls), and can be included both in the first and in the second groups. Similar results were obtained also for points $\mathrm{C} 2, \mathrm{~L} 1, \mathrm{~L} 8$ and $\mathrm{R} 1$, which are the ecotone areas between associations from the tunnel vault and walls. That is why their inclusion into one or another group achieved only the aim of increasing the number of analyzed species.

In the tunnel vault group, where, in addition to the vault itself, the points $\mathrm{L} 1$ and $\mathrm{R} 1$ were included, three associations (factors) can be clearly distinguished: (1) 4 amphipod species and barnacle $J$. marmorata, C. cristibrachium, Caprella neglecta, Pleusymtes sp., and Balanus crenatus; (2) hydroid, barnacle and amphipoda $O$. longissima, B. improvisus, and Gnorimosphaeroma ovatum; (3) two species of tubebuilding polychaetes and barnacle Potamilla sp., Hydroides ezoensis, and B. rostratus (Table 3). The three-factor solution was based, first of all on the results of the scree plot test (the plot contraflexure falls on the number of eigenvalue
Table 3. Factor loadings, total and percentage variance explained for biomass of some animal species occurring in association with the tunnel vault. Extraction method - principal components; factor rotation - varimax normalized.

\begin{tabular}{lrrr}
\hline \multicolumn{1}{c}{ Species } & \multicolumn{3}{c}{ Factor } \\
\cline { 2 - 4 } & \multicolumn{1}{c}{$\mathbf{1}$} & \multicolumn{1}{c}{$\mathbf{2}$} & \multicolumn{1}{c}{$\mathbf{3}$} \\
\hline Obelia longissima & 0.118 & $-\mathbf{0 . 9 6 1}$ & 0.137 \\
Hydroides ezoensis & 0.612 & $\mathbf{0 . 0 1 9}$ & $\mathbf{0 . 7 6 4}$ \\
Potamilla sp. & -0.002 & $\mathbf{0 . 0 1 8}$ & $\mathbf{0 . 9 8 3}$ \\
Balanus rostratus & -0.148 & 0.025 & $\mathbf{0 . 9 7 1}$ \\
Balanus improvisus & -0.088 & $-\mathbf{0 . 9 7 4}$ & -0.094 \\
Balanus crenatus & $\mathbf{0 . 8 4 0}$ & $\mathbf{0 . 1 0 0}$ & -0.100 \\
Gnorimosphaeroma ovatum & $-\mathbf{0 . 0 8 8}$ & $-\mathbf{0 . 9 7 4}$ & -0.094 \\
Jassa marmorata & $\mathbf{0 . 9 0 4}$ & -0.071 & 0.018 \\
Caprella cristibrachium & $\mathbf{0 . 9 5 8}$ & 0.010 & 0.074 \\
Caprella neglecta & $\mathbf{0 . 9 5 9}$ & 0.009 & 0.054 \\
Pleusymtes sp. & $\mathbf{0 . 9 4 6}$ & 0.037 & 0.031 \\
\hline Explained variance & 4.682 & 2.841 & 2.549 \\
\% of total explained variance & 0.426 & 0.258 & 0.23 \\
\hline Not Her
\end{tabular}

Note. Here and in Tables 4 and 5 factor loading $>0.7$ are in bold.

equal to 3, Fig. 6) and hierarchical analysis of the oblique factors: correlation coefficients of the initial clusters of 


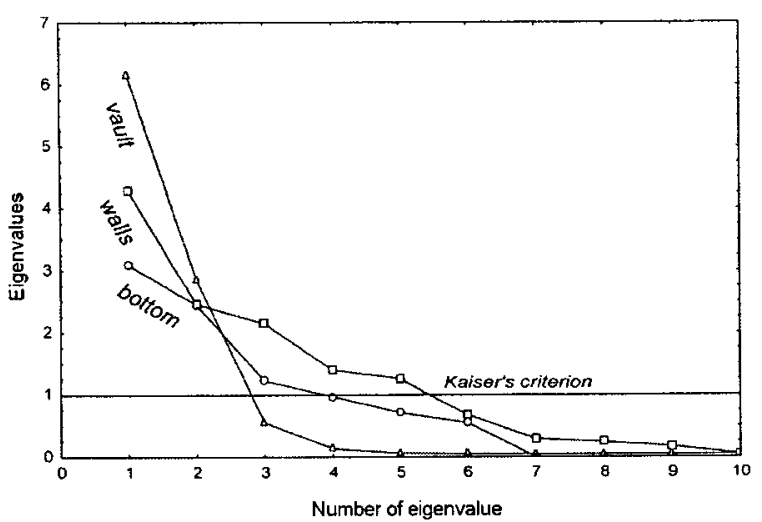

Fig. 6. Results of the scree plot test for factor solutions obtained when analyzing the structural interrelation for dominant and subdominant species, found in associations from different parts of the tunnel.

variables do not exceed 0.2 in all cases. The revealed factors explain in total more than $91 \%$ of biomass variance for separate animal species. Certain zonality can be traced to the distribution of the mentioned associations along the vault at the beginning of the tunnel: factor 2 maximum falls on point $\mathrm{C} 1$, factor 3 -on point $\mathrm{C} 2$, factor 1 -on point C3 (Fig. 7b).

In the group of foulers from the tunnel bottom, which includes only the samples collected from the bottom, the distributions of the most abundant species are substantially
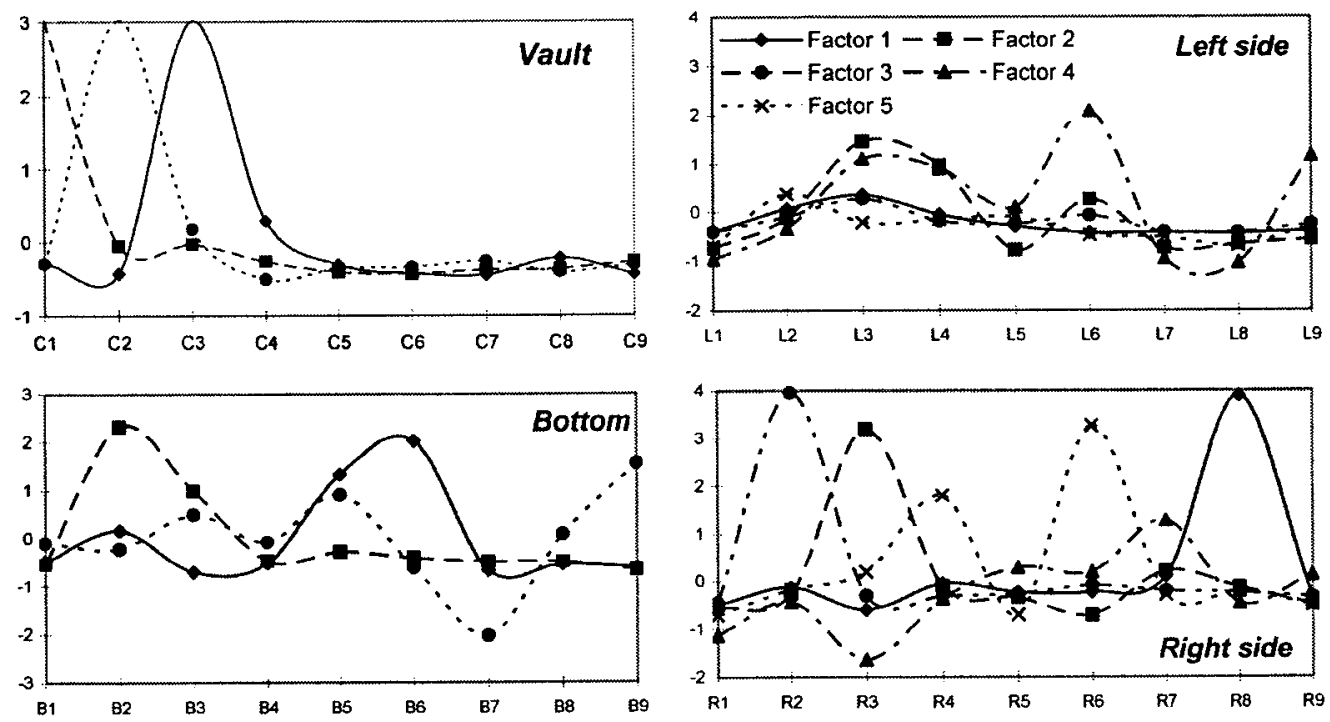

Fig. 7. Distribution of factor scores obtained when analyzing the biomass of fouling species from the different parts of the water intake tunnel of Vladivostok Heat and Power Plant. Factor scores for factor 2 (vault), factor 1 and 2 (bottom), and factor 1 and 3-5 (walls) were multiplied by -1 to obtain their positive values in points for the maximal biomass of species.

Table 4. Factor loadings, total and percentage variance explained for biomass of some animal species occurring in association with the tunnel bottom. Extraction method - principal components; factor rotation - varimax normalized.

\begin{tabular}{lrrr}
\hline \multirow{2}{*}{ Species } & \multicolumn{3}{c}{ Factor } \\
\cline { 2 - 4 } & \multicolumn{1}{c}{$\mathbf{1}$} & \multicolumn{1}{c}{$\mathbf{2}$} & \multicolumn{1}{c}{$\mathbf{3}$} \\
\hline Obelia longissima & 0.005 & $-\mathbf{0 . 9 9 7}$ & -0.017 \\
Hydroides ezoensis & $-\mathbf{0 . 9 7 7}$ & -0.161 & 0.080 \\
Potamilla sp. & $-\mathbf{0 . 9 8 0}$ & 0.117 & 0.034 \\
Balanus rostratus & 0.141 & 0.058 & $\mathbf{0 . 8 1 8}$ \\
Jassa marmorata & $-\mathbf{0 . 8 1 5}$ & 0.181 & 0.187 \\
Caprella cristibrachium & $\mathbf{0 . 1 8 8}$ & 0.229 & $-\mathbf{0 . 7 0 1}$ \\
Epheria turrita & -0.129 & $\mathbf{- 0 . 8 4 4}$ & 0.207 \\
Crenomytilus grayanus & -0.573 & 0.006 & -0.232 \\
Mytilus trossulus & 0.263 & $-\mathbf{0 . 7 6 5}$ & 0.006 \\
\hline Explained variance & 3.050 & 2.420 & 1.300 \\
\% of total explained variance & 0.339 & 0.269 & $\mathbf{0 . 1 4 4}$ \\
\hline
\end{tabular}

matched, and factor analysis methods clearly distinguish 2 associations: (1) 2 species of tube-building polychaetes and amphipoda $H$. ezoensis, Potamilla sp., and $J$. marmorata; (2) hydroid, gastropoda and mussel $O$. longissima, $E$. turrita, and $M$. trossulus (factors 1 and 2, Table 4). Only $B$. rostratus, $C$. cristibrachium and $C$. grayanus are distributed apart from those species. In this way, the distribution of C. grayanus is closely connected to the first 
Table 5. Factor loadings, total and percentage variance explained for biomass of some animal species occurring in association with the tunnel walls. Extraction method - principal components; factor rotation - varimax normalized.

\begin{tabular}{|c|c|c|c|c|c|}
\hline \multirow{2}{*}{ Species } & \multicolumn{5}{|c|}{ Factor } \\
\hline & 1 & 2 & 3 & 4 & 5 \\
\hline Obelia longissima & -0.970 & -0.008 & 0.051 & 0.116 & 0.051 \\
\hline Hydroides ezoensis & -0.562 & 0.402 & -0.116 & 0.067 & -0.600 \\
\hline Potamilla sp. & -0.364 & 0.812 & 0.085 & -0.165 & -0.025 \\
\hline Balanus rostratus & -0.236 & 0.710 & 0.174 & -0.482 & 0.045 \\
\hline Balanus improvisus & 0.107 & -0.137 & 0.117 & -0.029 & -0.933 \\
\hline Jassa marmorata & 0.186 & 0.834 & 0.072 & 0.400 & 0.015 \\
\hline Caprella cristibrachium & -0.831 & 0.490 & -0.070 & 0.014 & 0.021 \\
\hline Callista breviphonata & 0.153 & -0.070 & 0.124 & -0.697 & -0.239 \\
\hline Callithaca adamsi & 0.038 & -0.021 & -0.989 & 0.098 & 0.050 \\
\hline Hiatella arctica & -0.970 & 0.035 & 0.086 & 0.038 & 0.019 \\
\hline Mya japonica & 0.084 & 0.272 & 0.067 & -0.846 & 0.273 \\
\hline Mytilus trossulus & 0.034 & -0.046 & -0.988 & 0.098 & 0.033 \\
\hline Bugula pacifica & -0.079 & 0.949 & -0.126 & -0.098 & 0.051 \\
\hline Explained variance & 3.161 & 3.262 & 2.077 & 1.670 & 1.374 \\
\hline$\%$ of total explained variance & 0.243 & 0.251 & 0.160 & 0.128 & 0.106 \\
\hline
\end{tabular}

group, and variations in barnacle and caprellids biomass are in an antiphase state, forming the third factor. These three factors in total explain more than $75 \%$ of variance of the analyzed species biomass. Their selection is based on the results of hierarchical analysis of oblique factors ( $r<0.2$ for all cases), those of the scree plot test and the application of Kaiser criterion (Fig. 6). Distribution of factor values along the tunnel bottom is characterized by a clear separation of their maximums (Fig. 7a). Maximal values of factor 1 are observed in the middle of the tunnel, closer to its second turn (points B5 and B6); factor 2 before the first turn (B2), and factor 3-in the middle of the second turn (B7).

In the group of the tunnel walls, which includes only the samples from the walls, five such associations (factors) are found: (1) O. longissima, Hiatella arctica (bivalve), $C$. cristibrachium; (2) Bugula pacifica (bryozoa), J. marmorata, Potamilla sp., B. rostratus; (3) M. trossulus and Callithaca adamsi (bivalve); (4) Mya japonica and Callista breviphonata (bivalves); (5) B. improvisus and $H$. ezoensis (Table 5). The selection of the 5 factors, explaining in total $88.8 \%$ of the variable variance, were based on the consistency to the Kaiser criterion, results of scree plot test (Fig. 6) and hierarchical analysis $(r<0.32)$. As solutions, obtained by the principal component and maximum likelihood methods, coincided in details, $\chi^{2}$-criterion was used additionally. The maximal likelihood of the model was obtained precisely in the presence of these 5 factors. Distribution of factor scores along the tunnel walls is presented in Fig. 7 ( $c$ and d). As a whole, the patterns of these distributions at the right wall show more expressed maximums than those on the left, where they are more uniform, with some exceptions in the case of factor 4.

\section{Discussion}

Fouling organisms form the so-called "physically controlled" communities, which are typical for intertidal zones and estuaries, develop during 1-3 years and quite easily return to the initial state after stressful effects (Oshurkov 1994). For ships, such stressful effects, to a greater or lesser extent, involves a regular cleaning of hulls (Moshchenko and Zvyagintsev 2001a). That is why communities of ship foulers almost never reach a climactic state and exist in some succession stages, when relatively stable "fouling types" develop: communities of hydroids, barnacles, mussels, etc. A water intake tunnel community is also referred to as "physically controlled" communities, and the main factor, determining its state in a series of successionary transitions between "fouling types", is annual washing of the tunnel with hot water, which results in the deaths of most animals. That is why the tunnel communities are characterized by a relatively poor species composition. At the same time, a weak correlation between the biomass of separate species and total biomass indicates that during the period of its existence, the fouler community reaches such a level of succession, when animal distribution is mainly determined by biological interactions, but not a single (physical, chemical) 
controlling factor (Sukhanov et al. 1994).

A somewhat different picture can be seen when analyzing groups of different topological subdivisions in the tunnel. On the vault biomasses of dominant and subdominant species correlate highly with the total biomass, and, therefore, animal distribution is strictly controlled by a single factor. The degree and duration of drainage is evidently such a factor: tunnels of this type are never filled with water completely and their vault is mostly a zone of "uprush". Zonality of the tunnel vault is most probably also a result of the degree and duration of dewatering: at the opening of the tunnel, at almost permanent filling capacity, an unstable association to drainage species develops: $O$. longissima, $B$. improvisus and $G$. ovatum (section 1). Then the more drainage resistant association of Potamilla sp., B. rostratus and $H$. ezoensis (section 2 ) appears, and finally on the border of the permanent drainage-an association of $J$. marmorata, C. cristibrachium, C. neglecta, Pleusymtes sp. and $B$. crenatus (section 3).

Communities or fouling types, developed on the walls and bottom of the tunnel, are also "physically controlled" because of annual washing of the tunnel with hot water. But they are closer, than the vault group, to the "biologically balanced" communities of benthos, where biological relations of species are very diverse and in many respects determine their composition and structure. The presence of certain associations within walls and bottom groups, in which organisms are closely connected with each other, is sometimes determined by the fact, that one or several species use another or other species as a substrate, shelter or prey. For example, on the bottom of the tunnel shells of mussel $M$. trossulus serve as a substrate for settlement of $O$. longissima. Distribution of the snail $E$. turrita, which feeds on algae, is connected with spatial distribution of mussels, because the greatest number of algal fragments are observed exactly in the places of $M$. trossulus accumulations. O. longissima is a suitable substrate for $H$. arctica and $C$. cristibrachium on the tunnel walls. As a result, rather weak correlations are observed between the biomass of separate species and the total biomass.

In other cases, close matching of the distribution of different animal species is the result of a certain abiotic heterogeneity of the internal space of the tunnel and the appearance of areas favorable for the settling and dwelling of different foulers. It seems to explain the presence of associations of $J$. marmorata, Potamilla sp., B. rostratus

*Despite the existing opinion that caprellids feed on hydroids by observations of Marfenin (1993), they never eat them. (and B. pacifica, which uses calcareous shells of barnacles and polychaetes as a substrate), $M$. trossulus and $C$. adamsi, $M$. japonica and $C$. breviphonata, B. improvisus and $H$. ezoensis on the tunnel walls, and $H$. ezoensis, Potamilla sp. and $J$. marmorata along the bottom. It is also undoubtedly the case that this heterogeneity is responsible for the distinctions in distribution of substrate-forming species of the bottom and walls groups, such as $M$. trossulus, $B$. rostratus, and $O$. longissima.

Water movement is one of the basic factors determining animal distribution on natural and artificial substrates. On ships, for example, aggregated (contagious) distribution of foulers and their biomass variations are closely connected with the pattern of hull streamlining by water flow and formation of micro-scale turbulence zones in places of flow separation (Moshchenko and Zvyagintsev 2001b; Zvyagintsev and Moshchenko 2002). Despite a sufficiently simple geometry of the water intake tunnel, the water inside moves by quite a complicated pattem with formation of areas of local shear stress concentration, eddy zones and transverse circulations (Konstantinov et al. 1987; Moshchenko 2004). Therefore, the existence of spatially differentiated associations can be connected with hydrodynamic deformation of the water flow in the tunnel and with development of areas, favorable for the settling and dwelling of different substrate-forming fouler species first and foremost.

Vladivostok Heat and Power Plant has been in operation since 1980. In the course of its maintenance, the maximal damage was inflicted by mussel $M$. trossulus. In the beginning of its operation, mussel fouling developed along the full length of the tunnels, which could only be alleviated by mechanical means. By the end of March, 1987 in order to eradicate all fouling, thermal treatment of the tunnels was conducted using hot water. Heated seawater $\left(40-45^{\circ}\right)$ was pumped through the tunnel in an inverse direction for a total of 12 hours, which resulted in an almost total-lot effect: mussels survived only in the cracks.

Since 1987, thermal treatment of the tunnels has been performed regularly 3 times a year in the summertime, in June, July, and August. But thermal treatment is performed only in the tunnels. In pipes $400 \mathrm{~mm}$ in diameter, it cannot be applied for technical reasons, and the thickness of the long-surviving mussel communities in them has reached up to $150 \mathrm{~mm}$. Pipes with smaller diameter are completely choked by mussels with a shell length of about $24 \mathrm{~mm}$. On experimental plates, exposed in the water intake dipper of Vladivostok Heat and Power Plant, $M$. trossulus reached such lengths 6 months after settling (Zvyagintsev et al. 2004). Other methods of protection from fouling, chemical 
and physical, are not applied at the Vladivostok Heat and Power Plant.

In the area of Vladivostok Heat and Power Plant, as well as in its water intake dipper, the peak of $M$. trossulus larvae quantity in plankton falls in the first half of July, whereas in August larvae is absent in plankton (Zvyagintsev et al. 2004). On the basis of this data, thermal treatment of the tunnels has to be conducted in early August.

At the same time, the peak of juvenile mussel settling, up to $11500 \mathrm{ind} . \mathrm{m}^{2}$, with a shell length $<1 \mathrm{~mm}$, on plates in the water intake dipper of Vladivostok Heat and Power Plant at growing exposition time, falls on the second half of August. At that, mytilids settling happens later, up to the middle of November. It can be connected both with secondary, or post-larval settling of mussel juveniles, facilitated by a permanent one-directional water current, and with settling of larvae of other species in the Mytilidae family (it is difficult to identify juveniles that have settled very recently). Juvenile ability to become unfastened from substrate and to change initial biotope due to drift in water is a characteristic feature of mytilids, which is natural for M. trossulus as well (Brykov et al. 2000). Thus, thermal treatment, conducted in June, July and August, is not very effective.

M. trossulus larvae are irregularly distributed in water both vertically and horizontally (Zvyagintsev et al. 2004). On the stages, proceeding settling, their maximal concentration on the stations with depths no greater than $12 \mathrm{~m}$ can be observed in the upper $5 \mathrm{~m}$ range, and can reach 220 ind. $/ \mathrm{m}^{3}$. Below this depth, in $5-15 \mathrm{~m}$ layer, larvae quantity is sharply reduced to tens of individuals per cubic meter. At the station, located farther from the edge (22 m depth), larvae density at $0-15 \mathrm{~m}$ depth is not great and approximately the same, and in layers of $15-20 \mathrm{~m}$, it is reduced again more than twice. Thus, in order to reduce the intensity of settling of fouler larvae, it is recommended that the intake pipes of the cooling system be placed deeper than $15 \mathrm{~m}$, since when taking water from the surface, even insignificant density of larvae results in their considerable settling. It is evident that for $M$. trossulus larvae, concentration of 150-200 ind. $\mathrm{m}^{3}$ in water scoop plankton exceeds the rated value, as for the other mass foulers - $P$. limicola, $B$. crenatus, $B$. improvisus - larvae density even within the limits of $30-50$ ind. $/ \mathrm{m}^{3}$ causes mass fouling in the intake pipes.

\section{Acknowledgements}

The research described in this publication was made possible in part by the Russian Foundation for Basic Research (project no. 03-04-49573), Presidium of FEB RAS (grant "Marine biota response to environmental and climate change", 2004). Authors are sincerely grateful to all experts of the Institute of Marine Biology FEB RAS, who participated in identification of our materials, and to the workers of Vladivostok Heat and Power Plant for their assistance in collecting the material and for the provision of information related to the cooling system. Also, we wish to thank all anonymous reviewers for their kind and constructive comments, which helped us enhance our work to the optimal level.

\section{References}

Afifi, A. and C. Eisen. 1982. A statistical analysis: The approach with usage of computer. Mir, Moscow. 488 p. (In Russian)

Borovikov, V.P. and I.P. Borovikov. 1998. STATISTICA ${ }^{(B)}$ Statistical analysis and data processing in Windows ${ }^{(i B}$ media. Information and publishing house "Filin", Moscow. 608 p. (In Russian)

Brykov, V.A., O. Ya. Semenikhina, and N.K. Kolotukhina. 2000. Quantity dynamics of Mytilus trossulus mussel larvae in plankton and their settling on collectors in the Vostok Bay, the Sea of Japan. Rus. J. Mar. Biol., 26(4), 248-253.

Elliott, J.M. 1977. Some methods for the statistical analysis of samples of benthic invertebrates. Freshwater Biol. Ass. Sci. Publ., 25, 1-156.

Kim, J., U.P. Klekka, and C.U. Muller. 1989. Factor, discriminant and cluster analysis. Financy i statistika, Moscow. 312 p. (In Russian)

Kiyono, M. 2003. Control of biofouling in power plant cooling water systems-Discussion of practical research targets from a user's view. Sessile Organisms, 20(1), 11-13.

Konstantinov, N.M., N.A. Petrov, and L.I. Vysotsky. 1987. Hydraulics. Hydrology. Hydrometry. Part 1. General principles. Vysshaja shkola, Moscow. 304 p. (In Russian)

Maksimovich, N.V. and V.B. Pogrebov. 1986. The analysis of quantitative hydrobiological materials. LGU publishing house, Leningrad. 97 p. (In Russian)

Marfenin, N.N. 1993. The phenomenon of coloniality. MGU publishing house, Moscow. 239 p. (In Russian)

Moshchenko, A.V. 2004. The role of micro-scale turbulence in distribution and variability of benthic animals. Ph.D. Thesis, Institute of Marine Biology Vladivostok. 452 p. (In Russian)

Moshchenko, A.V. and A.Yu. Zvyagintsev. 2001a. Composition and structure of macrofouling communities on ocean-going ships in the Far East Sea Basin. Ocean Polar Res., 23(2), 63-75.

Moshchenko, A.V. and A.Yu. Zvyagintsev. 2001b. Features of the distribution of macrofouling organisms on the 
hulls of ocean-going ships. Ocean Polar Res., 23(4), 323-335.

Nosov, V.N. 1990. Principle components method and factor analysis. p. 162-187. In: Computer biometrics. ed. by V.N. Nosov. MGU Publishing House, Moscow. (In Russian)

Oshurkov, V.V. 1994. Succession of epibenthic communities by forming new substrates. Rus. J. Mar. Biol., 16(4), 247-251.

Propp, M.V. 1971. Ecology of coastal bottom communities of Murmansk coasts of the Barents Sea. Nauka, Moscow, 128 p. (In Russian)

Protasov, A.A., O.A. Sergeeva, S.I. Kosheleva, O.G. Kaftannikova, L.G. Lenchina, R.A. Kalinichenko, T.A. Vinogradskaya, B.I. Novikov, C.A. Afanasiev, and O.O. Sinitsina. 1991. Hydrobiology of reservoirs-coolers of thermal and nuclear plants. Naukova Dumka, Kiev. 192 p. (In Russian)

Reznichenko, O.G. 1978. Classification and spatio-scaled characteristic of fouling biotopes. Rus. J. Mar. Biol, 4(4), 3-15.

Sakaguchi, 1. 2003. An overview of the antifouling technologies in power plant cooling water systems. Sessile Organisms, 20(1), 15-19.

Sergy, G.A. and J.W. Evans. 1975. The settlement and distribution of marine organisms fouling in a seawater pipe system. Veliger, 18(1), 87-92.
Sudsuki, H. and K. Konne. 1970. Basic studies on the antifouling by ultrasonic waves for ship's bottom fouling organisms. J. Tokyo Univ. Fish., 5-6(1-2), 31-48.

Sukhanov, V.V., B.S. Petropavlovsky, and N.A. Chavtur. 1994. Structure of plant communities of Sikhote-Alin reserve. Dalnauka, Vladivostok. 220 p. (In Russian)

Turpaeva, E.P. 1987. Biological pattern of fouling community. Publishing house of the Institute of Oceanology, Moscow. 126 p. (In Russian)

Zevina, G.B. 1994. Biology of sea fouling. MGU Publishing House, Moscow. 133 p. (In Russian)

Zvyagintsev, A.Yu. and L.L. Budnikova. 2003. Amphipods (Amphipoda, Crustacea) in fouling of the cooling system of Vladivostok Heat and Power Plant \# 2. Izvestiya TINRO, 132, 280-298. (In Russian)

Zvyagintsev, A.Yu., O.M. Kom, and V.A. Kulikova. 2004. Seasonal dynamics of pelagic larvae and settling of fouling organisms in the conditions of hydrothermal pollution. Rus. J. Mar. Biol., 30(4), 296-307.

Zvyagintsev, A.Yu. and A.V. Moshchenko. 2002. The role of micro-scale turbulence in distribution of macro-fouling organisms on ocean-going ships. Rus. J. Mar. Biol., 28(6), 449-453. 